Article

\title{
More General Weighted-Type Fractional Integral Inequalities via Chebyshev Functionals
}

\author{
Gauhar Rahman ${ }^{1}$, Arshad Hussain ${ }^{1}$, Asad Ali ${ }^{1}$, Kottakkaran Sooppy Nisar ${ }^{2} * * \mathbb{D}$ and Roshan Noor Mohamed ${ }^{3}$ \\ 1 Department of Mathematics and Statistics, Hazara University, Mansehra 21300, Pakistan; \\ gauhar55uom@gmail.com or drgauhar.rahman@hu.edu.pk (G.R.); \\ arshadhussainpk224466@gmail.com (A.H.); asad_maths@hu.edu.pk (A.A.) \\ 2 Department of Mathematics, College of Arts and Sciences, Prince Sattam bin Abdulaziz University, \\ Wadi Aldawser 11991, Saudi Arabia \\ 3 Department of Pediatric Dentistry, Faculty of Dentistry, Taif University, P.O. Box 11099, \\ Taif 21944, Saudi Arabia; roshan.noor@tudent.edu.sa \\ * Correspondence: n.sooppy@psau.edu.sa or ksnisar1@gmail.com
}

check for updates

Citation: Rahman, G.; Hussain, A.; Ali, A.; Nisar, K.S.; Mohamed, R.N. More General Weighted-Type Fractional Integral Inequalities via Chebyshev Functionals. Fractal Fract. 2021, 5, 232. https://doi.org/ $10.3390 /$ fractalfract 5040232

Academic Editor: Norbert Herencsar

Received: 11 October 2021

Accepted: 16 November 2021

Published: 18 November 2021

Publisher's Note: MDPI stays neutral with regard to jurisdictional claims in published maps and institutional affiliations.

Copyright: (c) 2021 by the authors. Licensee MDPI, Basel, Switzerland. This article is an open access article distributed under the terms and conditions of the Creative Commons Attribution (CC BY) license (https:/ / creativecommons.org/licenses/by/ $4.0 /)$.

\begin{abstract}
The purpose of this research paper is first to propose the generalized weighted-type fractional integrals. Then, we investigate some novel inequalities for a class of differentiable functions related to Chebyshev's functionals by utilizing the proposed modified weighted-type fractional integral incorporating another function in the kernel $\mathcal{F}(\theta)$. For the weighted and extended Chebyshev's functionals, we also propose weighted fractional integral inequalities. With specific choices of $\omega(\theta)$ and $\mathcal{F}(\theta)$ as stated in the literature, one may easily study certain new inequalities involving all other types of weighted fractional integrals related to Chebyshev's functionals. Furthermore, the inequalities for all other type of fractional integrals associated with Chebyshev's functionals with certain choices of $\mathcal{\omega}(\theta)$ and $\mathcal{F}(\theta)$ are covered from the obtained generalized weighted-type fractional integral inequalities.
\end{abstract}

Keywords: Chebyshev's functional; inequalities; fractional integral; weighted fractional integral

MSC: 26D10; 26D15; 26D10; 26D53; 05A30

\section{Introduction}

In [1], for two integrable functions $\mathcal{Z}_{1}$ and $\mathcal{Z}_{2}$ on $\left[v_{1}, v_{2}\right]$, the Chebyshev functional and the weighted Chebyshev functional are respectively proposed as:

$$
T\left(\mathcal{Z}_{1}, \mathcal{Z}_{2}\right)=\frac{1}{v_{1}-v_{2}} \int_{v_{1}}^{v_{2}} \mathcal{Z}_{1}(\varrho) \mathcal{Z}_{2}(\varrho) d \varrho-\frac{1}{v_{1}-v_{2}}\left(\int_{v_{1}}^{v_{2}} \mathcal{Z}_{1}(\varrho) d \varrho\right) \frac{1}{v_{1}-v_{2}}\left(\int_{v_{1}}^{v_{2}} \mathcal{Z}_{2}(\varrho) d \varrho\right)
$$

and:

$$
T\left(\mathcal{Z}_{1}, \mathcal{Z}_{2}, \hbar_{1}\right)=\int_{v_{1}}^{v_{2}} \hbar_{1}(\varrho) d \varrho \int_{v_{1}}^{v_{2}} \hbar_{1}(\varrho) \mathcal{Z}_{1}(\varrho) \mathcal{Z}_{2}(\varrho) d \varrho-\int_{v_{1}}^{v_{2}} \hbar_{1}(\varrho) \mathcal{Z}_{1}(\varrho) d \varrho \int_{v_{1}}^{v_{2}} \hbar_{1}(\varrho) \mathcal{Z}_{2}(\varrho) d \varrho,
$$

where the function $\hbar_{1}$ is positive and integrable on $\left[v_{1}, v_{2}\right]$. In the study of probability and statistical problems, (2) has several applications. In addition, the functional (2) has applications in the domain of integral and differential equations. Readers may refer to [2-4].

For two differentiable functions $\mathcal{Z}_{1}$ and $\mathcal{Z}_{2}$, Dragomir [5] defined the inequality below as:

$$
\left|T\left(\mathcal{Z}_{1}, \mathcal{Z}_{2}, \hbar_{1}\right)\right| \leq\left\|\mathcal{Z}_{1}^{\prime}\right\|\left\|\mathcal{Z}_{2}^{\prime}\right\|\left[\int_{v_{1}}^{v_{2}} \hbar_{1}(\varrho) d \varrho \int_{v_{1}}^{v_{2}} \varrho^{2} \hbar_{1}(\varrho) d \varrho-\left(\int_{v_{1}}^{v_{2}} \varrho \hbar_{1}(\varrho) d \varrho\right)^{2}\right],
$$


where $\mathcal{Z}_{1}^{\prime}, \mathcal{Z}_{2}^{\prime} \in L_{\infty}\left(v_{1}, v_{2}\right)$ and $\hbar_{1}$ is integrable and a positive function on $\left[v_{1}, v_{2}\right]$. Using various methodologies, the researchers investigated the functionals (1) and (2) and discovered some notable inequalities. Readers are advised to see the works of [6-12]. Very recently, Srivastava et al. [13] investigated the Chebyshev inequality via the general family of fractional integral operators.

Elezovic et al. [14] proposed the inequality below for the weighted Chebyshev functional:

$$
\begin{aligned}
& \left|T\left(\mathcal{Z}_{1}, \mathcal{Z}_{2}, \hbar_{1}\right)\right| \leq \frac{1}{2}\left(\left.\int_{v_{1}}^{v_{2}} \int_{v_{1}}^{v_{2}} \hbar_{1}(\xi) \hbar_{1}(\zeta)|\xi-\zeta|^{\frac{1}{p^{\prime}}+\frac{1}{q^{\prime}}}\left|\int_{\zeta}^{\tau}\right| \mathcal{Z}_{1}^{\prime}(\varrho)\right|^{p} d \varrho \mid \frac{r}{p} d \xi d \zeta\right)^{\frac{1}{r}} \\
& \times\left(\left.\left.\int_{v_{1}}^{v_{2}} \int_{v_{1}}^{v_{2}} \hbar_{1}(\xi) \hbar_{1}(\zeta)|\xi-\zeta|^{\frac{1}{p^{\prime}}+\frac{1}{q^{\prime}}}\left|\int_{\zeta}^{\tau}\right| \mathcal{Z}_{2}^{\prime}(\varrho)\right|^{q} d \varrho\right|^{\frac{r}{q}} d \zeta d \zeta\right)^{\frac{1}{r^{\prime}}} \\
& \leq \frac{1}{2}\left\|\mathcal{Z}_{1}^{\prime}\right\|\left\|\mathcal{Z}_{2}^{\prime}\right\|\left(\int_{v_{1}}^{v_{2}} \int_{v_{1}}^{v_{2}} \hbar_{1}(\xi) \hbar_{1}(\zeta)|\xi-\zeta|^{\frac{1}{p^{\prime}}+\frac{1}{q^{\prime}}} d \xi d \zeta\right),
\end{aligned}
$$

where $\mathcal{Z}_{1}^{\prime} \in L^{p}\left(\left[v_{1}, v_{2}\right]\right), \mathcal{Z}_{2}^{\prime} \in L^{q}\left(\left[v_{1}, v_{2}\right]\right), p, q, r>1, \frac{1}{p}+\frac{1}{p^{\prime}}=1, \frac{1}{q}+\frac{1}{q^{\prime}}=1$, and $\frac{1}{r}+\frac{1}{r^{\prime}}=1$.

In [9], the authors established the following fractional integral inequality for the Chebyshev functional (2) by:

$$
\begin{aligned}
& 2\left|\mathcal{I}^{\alpha} \hbar_{1}(\tau) \mathcal{I}^{\alpha} \hbar_{1} \mathcal{Z}_{1} \mathcal{Z}_{2}(\theta)-\mathcal{I}^{\alpha} \hbar_{1} \mathcal{Z}_{1}(\theta) \mathcal{I}^{\alpha} \hbar_{1} \mathcal{Z}_{2}(\theta)\right| \\
\leq & \frac{\left\|\mathcal{Z}_{1}^{\prime}\right\|_{p}\left\|\mathcal{Z}_{2}^{\prime}\right\|_{q}}{\Gamma^{2}(\alpha)} \int_{0}^{\theta} \int_{0}^{\theta}(\theta-\varrho)^{\alpha-1}(\theta-\zeta)^{\alpha-1}|\varrho-\zeta| \hbar_{1}(\varrho) \hbar_{1}(\zeta) d \varrho d \zeta,
\end{aligned}
$$

where $\mathcal{Z}_{1}^{\prime} \in L^{p}\left(\left[0, \infty[), \mathcal{Z}_{2}^{\prime} \in L^{q}\left(\left[0, \infty[), p, q>1, \frac{1}{p}+\frac{1}{q}=1\right.\right.\right.\right.$.

In $[15,16]$, the extended Chebyshev functional was presented as:

$$
\begin{array}{r}
\tilde{T}\left(\mathcal{Z}_{1}, \mathcal{Z}_{2}, \hbar_{1}, \hbar_{1}^{\prime}\right)=\int_{v_{1}}^{v_{2}} \hbar_{1}^{\prime}(\varrho) d \varrho \int_{v_{1}}^{v_{2}} \hbar_{1}(\varrho) \mathcal{Z}_{1}(\varrho) \mathcal{Z}_{2}(\varrho) d \varrho+\int_{v_{1}}^{v_{2}} \hbar_{1}(\varrho) d \varrho \int_{v_{1}}^{v_{2}} \hbar_{1}^{\prime}(\varrho) \mathcal{Z}_{1}(\varrho) \mathcal{Z}_{2}(\varrho) d \varrho \\
-\int_{v_{1}}^{v_{2}} \hbar_{1}(\varrho) \mathcal{Z}_{1}(\varrho) d \varrho \int_{v_{1}}^{v_{2}} \hbar_{1}^{\prime}(\varrho) \mathcal{Z}_{2}(\varrho) d \varrho-\int_{v_{1}}^{v_{2}} \hbar_{1}^{\prime}(\varrho) \mathcal{Z}_{1}(\varrho) d \varrho \int_{v_{1}}^{v_{2}} \hbar_{1}(\varrho) \mathcal{Z}_{2}(\varrho) d \varrho .
\end{array}
$$

This paper is organized as follows:

The generalized weighted-type fractional integral inequalities connected to the functionals (1) and (2) are discussed in Section 2. We propose some generalized weighted-type fractional integral inequalities connected to (3) and (4) in Section 3. Finally, in Section 4, we give the concluding remarks.

We recall the following results from [17] as follows:

Definition 1. Suppose that the function $\Psi:[0, \infty) \rightarrow[0, \infty)$ satisfies the conditions given below:

$$
\begin{gathered}
\int_{0}^{1} \frac{\Psi(\varrho)}{\varrho} d \varrho, \\
\frac{1}{P} \leq \frac{\Psi(\mu)}{\Psi(v)} \leq P, \frac{1}{2} \leq \frac{\mu}{v} \leq 2, \\
\frac{\Psi(v)}{v^{2}} \leq Q \frac{\Psi(\mu)}{\mu^{2}}, \mu \leq v, \\
\left|\frac{\Psi(v)}{v^{2}}-\frac{\Psi(\mu)}{\mu^{2}}\right| \leq S|v-\mu| \frac{\Psi(v)}{v^{2}}, \frac{1}{2} \leq \frac{\mu}{v} \leq 2,
\end{gathered}
$$

where $P, Q, S>0$, independent of $\mu, v>0$. If $\Psi(v) v^{\alpha}$ is increasing for some $\alpha>0$ and $\frac{\Psi(v)}{v^{\beta}}$ is decreasing for some $\beta>0$, then $\Psi$ satisfies (5)-(8). 
Here, we define the following generalized weighted-type fractional integral operators.

Definition 2. The generalized weighted-type fractional integral operators, both left and right sided, are respectively defined by:

$$
\left({ }_{\omega}^{\mathcal{F}} \mathcal{I}_{v_{1}+}^{\Psi} \mathcal{Z}_{1}\right)(\theta)=\omega^{-1}(\theta) \int_{v_{1}}^{\theta} \frac{\Psi(\mathcal{F}(\theta)-\mathcal{F}(\varrho))}{\mathcal{F}(\theta)-\mathcal{F}(\varrho)} \omega(\varrho) \mathcal{F}^{\prime}(\varrho) \mathcal{Z}_{1}(\varrho) d \varrho, v_{1}<\theta,
$$

and:

$$
\left({ }_{\omega}^{\mathcal{F}} \mathcal{I}_{v_{2}}^{\Psi} \mathcal{Z}_{1}\right)(\theta)=\omega^{-1}(\theta) \int_{\theta}^{v_{2}} \frac{\Psi(\mathcal{F}(\varrho)-\mathcal{F}(\theta))}{\mathcal{F}(\varrho)-\mathcal{F}(\theta)} \omega(\varrho) \mathcal{F}^{\prime}(\varrho) \mathcal{Z}_{1}(\varrho) d \varrho, v_{2}>\theta .
$$

Remark 1. 1. If we consider $\Psi(\mathcal{F}(\theta))=\mathcal{F}(\theta)$, the fractional integrals (9) and (10) reduce to the following:

$$
\left({ }_{\omega}^{\mathcal{F}} \mathcal{I}_{v_{1}+} \mathcal{Z}_{1}\right)(\theta)=\omega^{-1}(\theta) \int_{v_{1}}^{\theta} \omega(\varrho) \mathcal{F}^{\prime}(\varrho) \mathcal{Z}_{1}(\varrho) d \varrho, v_{1}<\theta,
$$

and:

$$
\left({ }_{\omega}^{\mathcal{F}} \mathcal{I}_{v_{2}-} \mathcal{Z}_{1}\right)(\theta)=\omega^{-1}(\theta) \int_{\theta}^{v_{2}} \omega(\varrho) \mathcal{F}^{\prime}(\varrho) \mathcal{Z}_{1}(\varrho) d \varrho, v_{2}>\theta,
$$

respectively.

2. If we consider $\mathcal{F}(\theta)=\theta$, the fractional integrals (9) and (10) reduce to the following, respectively:

$$
\left({ }_{\omega}^{\mathcal{F}} \mathcal{I}_{v_{1}+} \mathcal{Z}_{1}\right)(\theta)=\mathfrak{\omega}^{-1}(\theta) \int_{v_{1}}^{\theta} \frac{\Psi(\theta-\varrho)}{\theta-\varrho} \omega(\varrho) \mathcal{Z}_{1}(\varrho) d \varrho, v_{1}<\theta,
$$

and:

$$
\left({ }_{\omega}^{\mathcal{F}} \mathcal{I}_{v_{2}-} \mathcal{Z}_{1}\right)(\theta)=\omega^{-1}(\theta) \int_{\theta}^{v_{2}} \frac{\Psi(\varrho-\theta)}{\varrho-\theta} \omega(\varrho) \mathcal{Z}_{1}(\varrho) d \varrho, v_{2}>\theta
$$

3. If we consider $\Psi(\mathcal{F}(\theta))=\frac{\mathcal{F}(\theta)^{\kappa}}{\Gamma(\kappa)}$, the fractional integrals (9) and (10) reduce to the following, respectively (see [18]):

$$
\left({ }_{\mathscr{\omega}}^{\mathcal{F}} \mathcal{I}_{v_{1}}^{\kappa} \mathcal{Z}_{1}\right)(\theta)=\frac{\mathscr{\omega}^{-1}(\theta)}{\Gamma(\kappa)} \int_{v_{1}}^{\theta}(\mathcal{F}(\theta)-\mathcal{F}(\varrho))^{\kappa-1} \varrho(\varrho) \mathcal{F}^{\prime}(\varrho) \mathcal{Z}_{1}(\varrho) d \varrho, v_{1}<\theta,
$$

and:

$$
\left({ }_{\omega}^{\mathcal{F}} \mathcal{I}_{v_{2}-}^{\kappa} \mathcal{Z}_{1}\right)(\theta)=\frac{\mathcal{\omega}^{-1}(\theta)}{\Gamma(\kappa)} \int_{\theta}^{v_{2}}(\mathcal{F}(\varrho)-\mathcal{F}(\theta))^{\kappa-1} \varrho(\varrho) \mathcal{F}^{\prime}(\varrho) \mathcal{Z}_{1}(\varrho) d \varrho, v_{2}>\theta,
$$

where $\kappa, \in \mathbb{C}$ with $\Re(\kappa)>0$.

4. If we consider $\mathcal{F}(\theta)=\theta$ and $\Psi(\mathcal{F}(\theta))=\frac{\theta^{\kappa}}{\Gamma(\kappa)}$, the fractional integrals (9) and (10) reduce to the following:

$$
\left(\omega^{\mathfrak{I}_{v_{1}}} \mathcal{Z}_{1}\right)(\theta)=\frac{\mathcal{\omega}^{-1}(\theta)}{\Gamma(\kappa)} \int_{v_{1}}^{\theta}(\theta-\varrho)^{\kappa-1} \mathfrak{\omega}(\varrho) \mathcal{Z}_{1}(\varrho) d \varrho, v_{1}<\theta,
$$

and:

$$
\left(\omega_{\omega} \mathfrak{I}_{v_{2}-}^{\kappa} \mathcal{Z}_{1}\right)(\theta)=\frac{\omega^{-1}(\theta)}{\Gamma(\kappa)} \int_{\theta}^{v_{2}}(\varrho-\theta)^{\kappa-1} \omega(\varrho) \mathcal{Z}_{1}(\varrho) d \varrho, v_{2}>\theta
$$


respectively.

5. If we consider $\mathcal{F}(\theta)=\ln \theta$ and $\Psi(\mathcal{F}(\theta))=\frac{(\ln \theta)^{\kappa}}{\Gamma(\kappa)}$, the fractional integrals (9) and (10) reduce to the following weighted Hadamard fractional integrals:

$$
\left(\omega_{\omega} \mathcal{I}_{v_{1}+}^{\kappa} \mathcal{Z}_{1}\right)(\theta)=\frac{\omega^{-1}(\theta)}{\Gamma(\kappa)} \int_{v_{1}}^{\theta}(\ln \theta-\ln \varrho)^{\kappa-1} \omega(\varrho) \mathcal{Z}_{1}(\varrho) \frac{d \varrho}{\varrho}, v_{1}<\theta,
$$

and:

$$
\left({ }_{\omega} \mathcal{I}_{v_{2}-}^{\kappa} \mathcal{Z}_{1}\right)(\theta)=\frac{\omega^{-1}(\theta)}{\Gamma(\kappa)} \int_{\theta}^{v_{2}}(\ln \varrho-\ln \theta)^{\kappa-1} \omega(\varrho) \mathcal{Z}_{1}(\varrho) \frac{d \varrho}{\varrho}, v_{2}>\theta .
$$

6. If we consider $\mathcal{F}(\theta)=\theta^{\eta}$ and $\Psi(\mathcal{F}(\theta))=\frac{\theta^{\eta}}{\eta}, \eta>0$, the fractional integrals (9) and (10) reduce to the following weighted Katugampola fractional integrals,

$$
\left({ }_{\omega} \mathcal{I}_{v_{1}}^{\kappa} \mathcal{Z}_{1}\right)(\theta)=\frac{\omega^{-1}(\theta)}{\Gamma(\kappa)} \int_{v_{1}}^{\theta}\left(\frac{\theta^{\eta}-\varrho^{\eta}}{\eta}\right)^{\kappa-1} \omega(\varrho) \mathcal{Z}_{1}(\varrho) \frac{d \varrho}{\varrho^{1-\eta}}, v_{1}<\theta
$$

and:

$$
\left({ }_{\omega} \mathcal{I}_{v_{2}}^{\kappa} \mathcal{Z}_{1}\right)(\theta)=\frac{\omega^{-1}(\theta)}{\Gamma(\kappa)} \int_{\theta}^{v_{2}}\left(\frac{\varrho^{\eta}-\theta^{\eta}}{\eta}\right)^{\kappa-1} \omega(\varrho) \mathcal{Z}_{1}(\varrho) \frac{d \varrho}{\varrho^{1-\eta}}, v_{2}>\theta
$$

7. If we consider $\mathcal{F}(\theta)=\theta$ and $\Psi(\mathcal{F}(\theta))=\frac{\theta}{\eta} \exp \left(-\frac{1-\eta}{\eta} \theta\right), \eta \in(0,1)$, the fractional integrals (9) and (10) reduce to the following weighted fractional integrals,

$$
\left({ }_{\omega} \mathcal{I}_{v_{1}+}^{\eta} \mathcal{Z}_{1}\right)(\theta)=\frac{\omega^{-1}(\theta)}{\eta} \int_{v_{1}}^{\theta} \exp \left(-\frac{1-\eta}{\eta}(\theta-\varrho)\right) \omega(\varrho) \mathcal{Z}_{1}(\varrho), v_{1}<\theta,
$$

and:

$$
\left({ }_{\omega} \mathcal{I}_{v_{2}-}^{\eta} \mathcal{Z}_{1}\right)(\theta)=\frac{\mathfrak{\omega}^{-1}(\theta)}{\eta} \int_{\theta}^{v_{2}} \exp \left(-\frac{1-\eta}{\eta}(\varrho-\theta)\right) \omega(\varrho) \mathcal{Z}_{1}(\varrho) d \varrho, v_{2}>\theta
$$

Furthermore, one can derive the weighted form of conformable fractional integrals introduced by [19-22].

The following special cases can be easily obtained by applying the conditions on $\omega(\theta)$ and $\Psi(\mathcal{F}(\theta))$.

Remark 2. 1. If we consider $\omega(\theta)=1$ and $\Psi(\mathcal{F}(\theta))=\mathcal{F}(\theta)$, the fractional integrals (9) and (10) reduce to the following:

$$
\left({ }^{\mathcal{F}} \mathcal{I}_{v_{1}+} \mathcal{Z}_{1}\right)(\theta)=\int_{v_{1}}^{\theta} \mathcal{F}^{\prime}(\varrho) \mathcal{Z}_{1}(\varrho) d \varrho, v_{1}<\theta,
$$

and:

$$
\left({ }^{\mathcal{F}} \mathcal{I}_{v_{2}-} \mathcal{Z}_{1}\right)(\theta)=\int_{\theta}^{v_{2}} \mathcal{F}^{\prime}(\varrho) \mathcal{Z}_{1}(\varrho) d \varrho, v_{2}>\theta
$$

respectively.

2. If we consider $\mathfrak{\omega}(\theta)=1$ and $\mathcal{F}(\theta)=\theta$, the fractional integrals (9) and (10) reduce to the following, respectively (see [23]):

$$
\left({ }^{\mathcal{F}} \mathcal{I}_{v_{1}+} \mathcal{Z}_{1}\right)(\theta)=\int_{v_{1}}^{\theta} \frac{\Psi(\theta-\varrho)}{\theta-\varrho} \mathcal{Z}_{1}(\varrho) d \varrho, v_{1}<\theta,
$$


and:

$$
\left({ }^{\mathcal{F}} \mathcal{I}_{v_{2}-} \mathcal{Z}_{1}\right)(\theta)=\int_{\theta}^{v_{2}} \frac{\Psi(\varrho-\theta)}{\varrho-\theta} \mathcal{Z}_{1}(\varrho) d \varrho, v_{2}>\theta
$$

3. If we consider $\omega(\theta)=1$ and $\Psi(\mathcal{F}(\theta))=\frac{\mathcal{F}(\theta)^{\kappa}}{\Gamma(\kappa)}$, the fractional integrals (9) and (10) reduce to the following, respectively (see $[24,25])$ :

$$
\left({ }^{\mathcal{F}} \mathcal{I}_{v_{1}}^{\kappa} \mathcal{Z}_{1}\right)(\theta)=\frac{1}{\Gamma(\kappa)} \int_{v_{1}}^{\theta}(\mathcal{F}(\theta)-\mathcal{F}(\varrho))^{\kappa-1} \mathcal{F}^{\prime}(\varrho) \mathcal{Z}_{1}(\varrho) d \varrho, v_{1}<\theta,
$$

and:

$$
\left({ }^{\mathcal{F}} \mathcal{I}_{v_{2}-}^{\kappa} \mathcal{Z}_{1}\right)(\theta)=\frac{1}{\Gamma(\kappa)} \int_{\theta}^{v_{2}}(\mathcal{F}(\varrho)-\mathcal{F}(\theta))^{\kappa-1} \mathcal{F}^{\prime}(\varrho) \mathcal{Z}_{1}(\varrho) d \varrho, v_{2}>\theta,
$$

where $\kappa, \in \mathbb{C}$ with $\Re(\kappa)>0$.

4. If we consider $\omega(\theta)=1, \mathcal{F}(\theta)=\theta$ and $\Psi(\mathcal{F}(\theta))=\frac{\theta^{\kappa}}{\Gamma(\kappa)}$, the fractional integrals (9) and (10) reduce to the following (see $[24,25])$ :

$$
\left(\mathfrak{I}_{v_{1}+}^{\kappa} \mathcal{Z}_{1}\right)(\theta)=\frac{1}{\Gamma(\kappa)} \int_{v_{1}}^{\theta}(\theta-\varrho)^{\kappa-1} \mathcal{Z}_{1}(\varrho) d \varrho, v_{1}<\theta,
$$

and:

$$
\left(\mathfrak{I}_{v_{2}-}^{\kappa} \mathcal{Z}_{1}\right)(\theta)=\frac{1}{\Gamma(\kappa)} \int_{\theta}^{v_{2}}(\varrho-\theta)^{\kappa-1} \mathcal{Z}_{1}(\varrho) d \varrho, v_{2}>\theta
$$

respectively.

5. If we consider $\omega(\theta)=1, \mathcal{F}(\theta)=\ln \theta$ and $\Psi(\mathcal{F}(\theta))=\frac{(\ln \theta)^{\kappa}}{\Gamma(\kappa)}$, the fractional integrals (9) and (10) reduce to the following weighted Hadamard fractional integrals (see $[24,25])$ :

$$
\left(\mathcal{I}_{v_{1}+}^{\kappa} \mathcal{Z}_{1}\right)(\theta)=\frac{1}{\Gamma(\kappa)} \int_{v_{1}}^{\theta}(\ln \theta-\ln \varrho)^{\kappa-1} \mathcal{Z}_{1}(\varrho) \frac{d \varrho}{\varrho}, v_{1}<\theta,
$$

and:

$$
\left(\mathcal{I}_{v_{2}-}^{\kappa} \mathcal{Z}_{1}\right)(\theta)=\frac{1}{\Gamma(\kappa)} \int_{\theta}^{v_{2}}(\ln \varrho-\ln \theta)^{\kappa-1} \mathcal{Z}_{1}(\varrho) \frac{d \varrho}{\varrho}, v_{2}>\theta
$$

6. If we consider $\omega(\theta)=1, \mathcal{F}(\theta)=\theta^{\eta}$, and $\Psi(\mathcal{F}(\theta))=\frac{\theta^{\eta}}{\eta}, \eta>0$, the fractional integrals (9) and (10) reduce to the following Katugampola [26] fractional integrals, respectively,

$$
\left(\mathcal{I}_{v_{1}}^{\kappa} \mathcal{Z}_{1}\right)(\theta)=\frac{1}{\Gamma(\kappa)} \int_{v_{1}}^{\theta}\left(\frac{\theta^{\eta}-\varrho^{\eta}}{\eta}\right)^{\kappa-1} \mathcal{Z}_{1}(\varrho) \frac{d \varrho}{\varrho^{1-\eta}}, v_{1}<\theta,
$$

and:

$$
\left(\mathcal{I}_{v_{2}}^{\kappa} \mathcal{Z}_{1}\right)(\theta)=\frac{1}{\Gamma(\kappa)} \int_{\theta}^{v_{2}}\left(\frac{\varrho^{\eta}-\theta^{\eta}}{\eta}\right)^{\kappa-1} \mathcal{Z}_{1}(\varrho) \frac{d \varrho}{\varrho^{1-\eta}}, v_{2}>\theta
$$

7. If we consider $\omega(\theta)=1, \mathcal{F}(\theta)=\theta$, and $\Psi(\mathcal{F}(\theta))=\frac{\theta}{\eta} \exp \left(-\frac{1-\eta}{\eta} \theta\right), \eta \in(0,1)$, the fractional integrals (9) and (10) reduce to the following weighted fractional integrals,

$$
\left(\mathcal{I}_{v_{1}+}^{\eta} \mathcal{Z}_{1}\right)(\theta)=\frac{1}{\eta} \int_{v_{1}}^{\theta} \exp \left(-\frac{1-\eta}{\eta}(\theta-\varrho)\right) \mathcal{Z}_{1}(\varrho), v_{1}<\theta,
$$


and:

$$
\left(\mathcal{I}_{v_{2}-}^{\eta} \mathcal{Z}_{1}\right)(\theta)=\frac{1}{\eta} \int_{\theta}^{v_{2}} \exp \left(-\frac{1-\eta}{\eta}(\varrho-\theta)\right) \mathcal{Z}_{1}(\varrho) d \varrho, v_{2}>\theta
$$

Similarly, (9) and (10) will lead to the fractional integrals defined by [19-22].

\section{Generalized Weighted-Type Fractional Integral Inequalities via Chebyshev's Functional}

Here, we develop weighted-type generalized fractional integral inequalities via Chebyshev's functional.

Theorem 1. If the two functions $\mathcal{Z}_{1}$ and $\mathcal{Z}_{2}$ are differentiable on $[0, \infty)$ with $\mathcal{Z}_{1}^{\prime}, \mathcal{Z}_{2}^{\prime} \in \mathcal{L}_{\infty}([0, \infty[)$ and we suppose $\mathcal{F}$ is positive and increasing on $[0, \infty[$ and its derivative is continuous on $[0, \infty[$, then the following inequality holds:

$$
\begin{aligned}
& \left|\left({ }_{\omega}^{\mathcal{F}} \mathcal{I}_{v_{1}+}^{\Psi} 1\right)(\theta)\left({ }^{\mathcal{F}} \mathcal{I}_{v_{1}+}^{\Psi} \mathcal{Z}_{1} \mathcal{Z}_{2}\right)(\theta)-\left({ }_{\omega}^{\mathcal{F}} \mathcal{I}_{v_{1}+}^{\Psi} \mathcal{Z}_{1}\right)(\theta)\left({ }_{\omega}^{\mathcal{F}} \mathcal{I}_{v_{1}+}^{\Psi} \mathcal{Z}_{2}(\theta)\right)\right| \\
\leq & \left\|\mathcal{Z}_{1}^{\prime}\right\|_{\infty}\left\|\mathcal{Z}_{2}^{\prime}\right\|_{\infty}\left[\left({ }_{{ }_{\omega}}^{\mathcal{F}} \mathcal{I}_{v_{1}+}^{\Psi} 1\right)(\theta)\left({ }_{\omega}^{\mathcal{F}} \mathcal{I}_{v_{1}+}^{\Psi} \theta^{2}\right)-\left(\left({ }_{\omega}^{\mathcal{F}} \mathcal{I}_{v_{1}+}^{\Psi} \theta\right)\right)^{2}\right],
\end{aligned}
$$

where $\left({ }_{\omega}^{\mathcal{F}} \mathcal{I}_{v_{1}+}^{\Psi} 1\right)(\theta)$ is defined by:

$$
\left({ }_{\omega}^{\mathcal{F}} \mathcal{I}_{v_{1}+}^{\Psi} 1\right)(\theta)=\omega^{-1}(\theta) \int_{v_{1}}^{\theta} \frac{\Psi(\mathcal{F}(\theta)-\mathcal{F}(\varrho))}{\mathcal{F}(\theta)-\mathcal{F}(\varrho)} \omega(\varrho) \mathcal{F}^{\prime}(\varrho) d \varrho, v_{1}<\theta
$$

Proof. Let us define:

$$
H(\varrho, \zeta)=\left(\mathcal{Z}_{1}(\varrho)-\mathcal{Z}_{1}(\zeta)\right)\left(\mathcal{Z}_{2}(\varrho)-\mathcal{Z}_{2}(\zeta)\right) ; \varrho, \zeta \in\left(v_{1} \theta\right) .
$$

The product of (12) by $\omega^{-1}(\theta) \frac{\Psi(\mathcal{F}(\theta)-\mathcal{F}(\varrho))}{\mathcal{F}(\theta)-\mathcal{F}(\varrho)} \varrho(\varrho) \mathcal{F}^{\prime}(\varrho)$ and then integrating with respect to $\varrho$ over $\left(v_{1}, \theta\right)$ and employing $(9)$, we have:

$$
\begin{aligned}
& \mathscr{\omega}^{-1}(\theta) \int_{v_{1}}^{\theta} \frac{\Psi(\mathcal{F}(\theta)-\mathcal{F}(\varrho))}{\mathcal{F}(\theta)-\mathcal{F}(\varrho)} \mathfrak{\omega}(\varrho) \mathcal{F}^{\prime}(\varrho) H(\varrho, \zeta) d \varrho \\
= & \left({ }_{\mathscr{\omega}}^{\mathcal{F}} \mathcal{I}_{v_{1}+}^{\Psi} \mathcal{Z}_{1} \mathcal{Z}_{2}\right)(\theta)-\mathcal{Z}_{1}(\zeta)\left({ }_{\omega}^{\mathcal{F}} \mathcal{I}_{v_{1}+}^{\Psi} \mathcal{Z}_{2}\right)(\theta)-\mathcal{Z}_{2}(\zeta)\left({ }_{\mathscr{\omega}}^{\mathcal{F}} \mathcal{I}_{v_{1}+}^{\Psi} \mathcal{Z}_{1}\right)(\theta)+\mathcal{Z}_{1}(\zeta) \mathcal{Z}_{2}(\zeta)\left({ }^{\mathcal{F}} \mathcal{I}_{v_{1}}^{\Psi} 1\right)(\theta) .
\end{aligned}
$$

Again, conducting the product (13) by $\omega^{-1}(\theta) \frac{\Psi(\mathcal{F}(\theta)-\mathcal{F}(\zeta))}{\mathcal{F}(\theta)-\mathcal{F}(\zeta)} \mathfrak{\omega}(\zeta) \mathcal{F}^{\prime}(\zeta)$ and then integrating with respect to $\zeta$ over $\left(v_{1}, \theta\right)$, we have:

$$
\begin{aligned}
& \omega^{-2}(\theta) \int_{v_{1}}^{\theta} \int_{v_{1}}^{\theta} \frac{\Psi(\mathcal{F}(\theta)-\mathcal{F}(\varrho))}{\mathcal{F}(\theta)-\mathcal{F}(\varrho)} \frac{\Psi(\mathcal{F}(\theta)-\mathcal{F}(\zeta))}{\mathcal{F}(\theta)-\mathcal{F}(\zeta)} \mathcal{F}^{\prime}(\varrho) \omega(\varrho) \omega(\zeta) \mathcal{F}^{\prime}(\zeta) H(\varrho, \zeta) d \varrho d \zeta \\
= & 2\left(\left({ }_{\mathscr{\omega}}^{\mathcal{F}} \mathcal{I}_{v_{1}+}^{\Psi} 1\right)(\theta)\left({ }^{\mathcal{F}} \mathcal{I}_{v_{1}+}^{\Psi} \mathcal{Z}_{1} \mathcal{Z}_{2}\right)(\theta)-\left({ }_{\mathscr{\omega}}^{\mathcal{F}} \mathcal{I}_{v_{1}+}^{\Psi} \mathcal{Z}_{1}\right)(\theta)\left({ }_{\mathscr{\omega}}^{\mathcal{F}} \mathcal{I}_{v_{1}+}^{\Psi} \mathcal{Z}_{2}\right)(\theta)\right) .
\end{aligned}
$$

On the other side, we also have:

$$
H(\varrho, \zeta)=\int_{\varrho}^{\zeta} \int_{\varrho}^{\zeta} \mathcal{Z}_{1}^{\prime}(x) \mathcal{Z}_{2}^{\prime}(y) d x d y
$$

Since $\mathcal{Z}_{1}^{\prime}(x), \mathcal{Z}_{2}^{\prime}(y) \in L_{\infty}([0, \infty[)$, therefore we have:

$$
|H(\varrho, \zeta)| \leq\left|\int_{\varrho}^{\zeta} \mathcal{Z}_{1}^{\prime}(x) d x\left\|\int_{\varrho}^{\zeta} \mathcal{Z}_{2}^{\prime}(y) d y \mid \leq\right\| \mathcal{Z}_{1}^{\prime}\left\|_{\infty}\right\| \mathcal{Z}_{2}^{\prime} \|_{\infty}(\varrho-\zeta)^{2} .\right.
$$


Therefore, it can be written as:

$$
\begin{aligned}
& \omega^{-2}(\theta) \int_{v_{1}}^{\theta} \int_{v_{1}}^{\theta} \frac{\Psi(\mathcal{F}(\theta)-\mathcal{F}(\varrho))}{\mathcal{F}(\theta)-\mathcal{F}(\varrho)} \frac{\Psi(\mathcal{F}(\theta)-\mathcal{F}(\zeta))}{\mathcal{F}(\theta)-\mathcal{F}(\zeta)} \mathcal{F}^{\prime}(\varrho) \omega(\varrho) \mathcal{F}^{\prime}(\zeta) \omega(\zeta)|H(\varrho, \zeta)| d \varrho d \zeta \\
\leq & \left\|\mathcal{Z}_{1}^{\prime}\right\|_{\infty}\left\|\mathcal{Z}_{2}^{\prime}\right\|_{\infty} \omega^{-2}(\theta) \int_{v_{1}}^{\theta} \int_{v_{1}}^{\theta} \frac{\Psi(\mathcal{F}(\theta)-\mathcal{F}(\varrho))}{\mathcal{F}(\theta)-\mathcal{F}(\varrho)} \frac{\Psi(\mathcal{F}(\theta)-\mathcal{F}(\zeta))}{\mathcal{F}(\theta)-\mathcal{F}(\zeta)} \mathcal{F}^{\prime}(\varrho) \omega(\varrho) \mathcal{F}^{\prime}(\zeta) \omega(\zeta) \\
\times & \left(\varrho^{2}-2 \varrho \zeta+\zeta^{2}\right) d \varrho d \zeta .
\end{aligned}
$$

From (17), we obtain:

$$
\begin{aligned}
& \boldsymbol{\omega}^{-2}(\theta) \int_{v_{1}}^{\theta} \int_{v_{1}}^{\theta} \frac{\Psi(\mathcal{F}(\theta)-\mathcal{F}(\varrho))}{\mathcal{F}(\theta)-\mathcal{F}(\varrho)} \frac{\Psi(\mathcal{F}(\theta)-\mathcal{F}(\zeta))}{\mathcal{F}(\theta)-\mathcal{F}(\zeta)} \mathcal{F}^{\prime}(\varrho) \omega(\varrho) \mathcal{F}^{\prime}(\zeta) \omega(\zeta)|H(\varrho, \zeta)| d \varrho d \zeta \\
\leq & 2\left\|\mathcal{Z}_{1}^{\prime}\right\|_{\infty}\left\|\mathcal{Z}_{2}^{\prime}\right\|_{\infty}\left[\left({ }^{\mathcal{F}} \mathcal{I}_{v_{1}}^{\Psi} 1\right)(\theta)\left({ }^{\mathcal{F}} \mathcal{I}_{v_{1}+}^{\Psi} \theta^{2}\right)-\left({ }^{\mathcal{F}} \mathcal{I}_{v_{1}+}^{\Psi} \theta\right)^{2}\right] .
\end{aligned}
$$

Hence, from (14) and (18), we obtain the required proof.

Corollary 1. If the two functions $\mathcal{Z}_{1}$ and $\mathcal{Z}_{2}$ are differentiable on $[0, \infty)$ with $\mathcal{Z}_{1}^{\prime}, \mathcal{Z}_{2}^{\prime} \in \mathcal{L}_{\infty}([0, \infty[)$ and we let $\mathcal{F}$ be a positive and increasing function on $[0, \infty[$ and its derivative be continuous on $[0, \infty[$, then the following inequality holds:

$$
\begin{aligned}
& \left|\Pi(1)\left({ }^{\mathcal{F}} \mathcal{I}_{v_{1}+}^{\Psi} \mathcal{Z}_{1} \mathcal{Z}_{2}\right)(\theta)-\left({ }^{\mathcal{F}} \mathcal{I}_{v_{1}+}^{\Psi} \mathcal{Z}_{1}\right)(\theta)\left({ }^{\mathcal{F}} \mathcal{I}_{v_{1}+}^{\Psi} \mathcal{Z}_{2}\right)(\theta)\right| \\
\leq & \left\|\mathcal{Z}_{1}^{\prime}\right\|_{\infty}\left\|\mathcal{Z}_{2}^{\prime}\right\|_{\infty}\left[\Pi(1)\left({ }^{\mathcal{F}} \mathcal{I}_{v_{1}+}^{\Psi} \theta^{2}\right)-\left({ }^{\mathcal{F}} \mathcal{I}_{v_{1}+}^{\Psi} \theta\right)^{2}\right],
\end{aligned}
$$

where $\Pi(1)$ is defined by:

$$
\Pi(1)=\int_{v_{1}}^{\theta} \frac{\Psi(\mathcal{F}(\theta)-\mathcal{F}(\varrho))}{\mathcal{F}(\theta)-\mathcal{F}(\varrho)} \mathcal{F}^{\prime}(\varrho) d \varrho
$$

Theorem 2. If the two functions $\mathcal{Z}_{1}$ and $\mathcal{Z}_{2}$ are differentiable, both have variations in same sense on $[0, \infty)$, and we let $\hbar_{1}$ be a positive function on $[0, \infty)$. Suppose that $\mathcal{F}$ is positive and increasing on $\left[0, \infty\left[\right.\right.$ and its derivative is continuous on $\left[0, \infty\left[\right.\right.$. Let $\mathcal{Z}_{1}^{\prime}, \mathcal{Z}_{2}^{\prime} \in \mathcal{L}_{\infty}([0, \infty[)$, then the following inequality holds:

$$
\begin{aligned}
& 0 \leq\left({ }_{\omega}^{\mathcal{F}} \mathcal{I}_{v_{1}+}^{\Psi} \hbar_{1}\right)(\theta)\left({ }_{\omega}^{\mathcal{F}} \mathcal{I}_{v_{1}+}^{\Psi} \hbar_{1} \mathcal{Z}_{1} \mathcal{Z}_{2}\right)(\theta)-\left({ }_{\omega}^{\mathcal{F}} \mathcal{I}_{v_{1}+}^{\Psi} \hbar_{1} \mathcal{Z}_{1}\right)(\theta)\left({ }_{\omega}^{\mathcal{F}} \mathcal{I}_{v_{1}+}^{\Psi} \hbar_{1} \mathcal{Z}_{2}\right)(\theta) \\
& \leq\left\|\mathcal{Z}_{1}^{\prime}\right\|_{\infty}\left\|\mathcal{Z}_{2}^{\prime}\right\|_{\infty}\left[\left({ }^{\mathcal{F}} \mathcal{I}_{v_{1}+}^{\Psi} \hbar_{1}\right)(\theta)\left({ }^{\mathcal{F}} \mathcal{I}_{v_{1}+}^{\Psi} \theta^{2} \hbar_{1}\right)(\theta)-\left({ }_{\omega}^{\mathcal{F}} \mathcal{I}_{0}^{\Psi} \theta \hbar_{1}\right)^{2}(\theta)\right] .
\end{aligned}
$$

Proof. Define:

$$
\begin{array}{r}
H(\varrho, \zeta)=\left(\mathcal{Z}_{1}(\varrho)-\mathcal{Z}_{1}(\zeta)\right)\left(\mathcal{Z}_{2}(\zeta)-\mathcal{Z}_{2}(\zeta)\right) ; \varrho, \zeta \in\left(v_{1} \theta\right), \theta>0 \\
\quad=\mathcal{Z}_{1}(\varrho) \mathcal{Z}_{2}(\varrho)-\mathcal{Z}_{1}(\varrho) \mathcal{Z}_{2}(\zeta)-\mathcal{Z}_{1}(\zeta) \mathcal{Z}_{2}(\varrho)+\mathcal{Z}_{1}(\zeta) \mathcal{Z}_{2}(\zeta)
\end{array}
$$

By Theorem 2, $\mathcal{Z}_{1}$ and $\mathcal{Z}_{2}$ fulfil the hypothesis; therefore, we have:

$$
H(\varrho, \zeta) \geq 0 .
$$


Taking the product on both sides of (20) by $\omega^{-1}(\theta) \frac{\Psi(\mathcal{F}(\theta)-\mathcal{F}(\varrho))}{\mathcal{F}(\theta)-\mathcal{F}(\varrho)} \omega(\varrho) \mathcal{F}^{\prime}(\varrho) \hbar_{1}(\varrho)$ and, then, taking the integration of both sides with respect to $\varrho$ over $\left(v_{1}, \theta\right)$ and:

$$
\begin{aligned}
& { }^{-1}(\theta) \int_{v_{1}}^{\theta} \frac{\Psi(\mathcal{F}(\theta)-\mathcal{F}(\varrho))}{\mathcal{F}(\theta)-\mathcal{F}(\varrho)} \omega(\varrho) \mathcal{F}^{\prime}(\varrho) \hbar_{1}(\varrho) H(\varrho, \zeta) d \varrho \\
= & \left({ }_{\omega}^{\mathcal{F}} \mathcal{I}_{v_{1}+}^{\Psi} \hbar_{1} \mathcal{Z}_{1} \mathcal{Z}_{2}\right)(\theta)-\mathcal{Z}_{2}(\zeta)\left({ }_{\omega}^{\mathcal{F}} \mathcal{I}_{v_{1}+}^{\Psi} \hbar_{1} \mathcal{Z}_{1}\right)(\theta)-\mathcal{Z}_{2}(\zeta)\left({ }_{\omega}^{\mathcal{F}} \mathcal{I}_{v_{1}+}^{\Psi} \hbar_{1} \mathcal{Z}_{2}\right)(\theta) \\
+ & \mathcal{Z}_{1}(\zeta) \mathcal{Z}_{2}(\zeta)\left({ }_{\omega}^{\mathcal{F}} \mathcal{I}_{v_{1}+}^{\Psi} \hbar_{1}\right)(\theta) \geq 0 .
\end{aligned}
$$

Again, taking the product of $(21)$ by $\omega^{-1}(\theta) \frac{\Psi(\mathcal{F}(\theta)-\mathcal{F}(\zeta))}{\mathcal{F}(\theta)-\mathcal{F}(\zeta)} \omega(\zeta) \mathcal{F}^{\prime}(\zeta) \hbar_{1}(\zeta)$, then taking integration with respect to $\zeta$ over $\left(v_{1}, \theta\right)$ and using (9), we obtain:

$$
\begin{aligned}
& \boldsymbol{\omega}^{-2}(\theta) \int_{v_{1}}^{\theta} \int_{v_{1}}^{\theta} \frac{\Psi(\mathcal{F}(\theta)-\mathcal{F}(\varrho))}{\mathcal{F}(\theta)-\mathcal{F}(\varrho)} \frac{\Psi(\mathcal{F}(\theta)-\mathcal{F}(\zeta))}{\mathcal{F}(\theta)-\mathcal{F}(\zeta)} \mathcal{F}^{\prime}(\varrho) \omega(\varrho) \mathcal{F}^{\prime}(\zeta) \omega(\zeta) \hbar_{1}(\varrho) \hbar_{1}(\zeta) H(\varrho, \zeta) d \varrho d \zeta \\
= & \left({ }_{\omega}^{\mathcal{F}} \mathcal{I}_{v_{1}+}^{\Psi} \hbar_{1}\right)(\theta)\left({ }_{\omega}^{\mathcal{F}} \mathcal{I}_{v_{1}+}^{\Psi} \hbar_{1} \mathcal{Z}_{1} \mathcal{Z}_{2}\right)(\theta)-\left({ }^{\mathcal{F}} \mathcal{I}_{v_{1}+}^{\Psi} \hbar_{1} \mathcal{Z}_{1}\right)(\theta)\left({ }_{\omega}^{\mathcal{F}} \mathcal{I}_{v_{1}+}^{\Psi} \hbar_{1} \mathcal{Z}_{2}\right)(\theta) \geq 0 .
\end{aligned}
$$

From (16), it becomes:

$$
\begin{aligned}
& \frac{\omega^{-2}(\theta)}{2} \int_{v_{1}}^{\theta} \int_{v_{1}}^{\theta} \frac{\Psi(\mathcal{F}(\theta)-\mathcal{F}(\varrho))}{\mathcal{F}(\theta)-\mathcal{F}(\varrho)} \frac{\Psi(\mathcal{F}(\theta)-\mathcal{F}(\zeta))}{\mathcal{F}(\theta)-\mathcal{F}(\zeta)} \mathcal{F}^{\prime}(\varrho) \omega(\varrho) \mathcal{F}^{\prime}(\zeta) \omega(\zeta) \hbar_{1}(\varrho) \hbar_{1}(\zeta)|H(\varrho, \zeta)| d \varrho d \zeta \\
\leq & \frac{\left\|\mathcal{Z}_{1}^{\prime}\right\|_{\infty}\left\|\mathcal{Z}_{2}^{\prime}\right\|_{\infty} \mathcal{\omega}^{-2}(\theta)}{2} \int_{v_{1}}^{\theta} \int_{v_{1}}^{\theta} \frac{\Psi(\mathcal{F}(\theta)-\mathcal{F}(\varrho))}{\mathcal{F}(\theta)-\mathcal{F}(\varrho)} \frac{\Psi(\mathcal{F}(\theta)-\mathcal{F}(\zeta))}{\mathcal{F}(\theta)-\mathcal{F}(\zeta)} \mathcal{F}^{\prime}(\varrho) \omega(\varrho) \mathcal{F}^{\prime}(\zeta) \omega(\zeta) \hbar_{1}(\varrho) \hbar_{1}(\zeta) \\
\times & \left(\varrho^{2}-2 \varrho \zeta+\zeta^{2}\right) d \varrho d \zeta .
\end{aligned}
$$

Consequently, it can be written as:

$$
\begin{aligned}
& \frac{\omega^{-2}(\theta)}{2} \int_{v_{1}}^{\theta} \int_{v_{1}}^{\theta} \frac{\Psi(\mathcal{F}(\theta)-\mathcal{F}(\varrho))}{\mathcal{F}(\theta)-\mathcal{F}(\varrho)} \frac{\Psi(\mathcal{F}(\theta)-\mathcal{F}(\zeta))}{\mathcal{F}(\theta)-\mathcal{F}(\zeta)} \mathcal{F}^{\prime}(\varrho) \omega(\varrho) \mathcal{F}^{\prime}(\zeta) \omega(\zeta) \hbar_{1}(\varrho) \hbar_{1}(\zeta)|H(\varrho, \zeta)| d \varrho d \zeta \\
\leq & 2\left\|\mathcal{Z}_{1}^{\prime}\right\|_{\infty}\left\|\mathcal{Z}_{2}^{\prime}\right\|_{\infty}\left[\left({ }^{\mathcal{F}} \mathcal{I}_{v_{1}+}^{\Psi} \hbar_{1}\right)(\theta)\left({ }_{\omega}^{\mathcal{F}} \mathcal{I}_{v_{1}+}^{\Psi} \theta^{2} \hbar_{1}\right)(\theta)-\left({ }_{\omega}^{\mathcal{F}} \mathcal{I}_{0}^{\kappa} \theta \hbar_{1}\right)^{2}(\theta)\right] .
\end{aligned}
$$

According to (22) and (24), we obtain the desired proof.

Setting Theorem 2 for $\mathfrak{O}=1$, we obtain the following new result.

Corollary 2. If the two functions $\mathcal{Z}_{1}$ and $\mathcal{Z}_{2}$ are differentiable, both have variations in the same sense on $[0, \infty)$ and $\hbar_{1}$ is a positive function on $[0, \infty)$. Suppose that $\mathcal{F}$ is a positive and increasing function on $\left[0, \infty\left[\right.\right.$ and its derivative is continuous on $\left[0, \infty\left[\right.\right.$. If $\mathcal{Z}_{1}^{\prime}, \mathcal{Z}_{2}^{\prime} \in \mathcal{L}_{\infty}([0, \infty[)$, then the following inequality holds:

$$
\begin{aligned}
& \quad 0 \leq\left({ }^{\mathcal{F}} \mathcal{I}_{v_{1}+}^{\Psi} \hbar_{1}\right)(\theta)\left({ }^{\mathcal{F}} \mathcal{I}_{v_{1}+}^{\Psi} \hbar_{1} \mathcal{Z}_{1} \mathcal{Z}_{2}\right)(\theta)-\left({ }^{\mathcal{F}} \mathcal{I}_{v_{1}+}^{\Psi} \hbar_{1} \mathcal{Z}_{1}\right)(\tau)\left({ }^{\mathcal{F}} \mathcal{I}_{v_{1}+}^{\Psi} \hbar_{1} \mathcal{Z}_{2}\right)(\theta) \\
& \leq\left\|\mathcal{Z}_{1}^{\prime}\right\|_{\infty}\left\|\mathcal{Z}_{2}^{\prime}\right\|_{\infty}\left[\left({ }^{\mathcal{F}} \mathcal{I}_{v_{1}+}^{\Psi} \hbar_{1}\right)(\theta)\left({ }^{\mathcal{F}} \mathcal{I}_{v_{1}+}^{\Psi} \theta^{2} \hbar_{1}\right)(\tau)-\left({ }^{\mathcal{F}} \mathcal{I}_{0}^{\kappa} \theta \hbar_{1}(\theta)\right)^{2}\right] .
\end{aligned}
$$

Remark 3. By considering $\hbar_{1}(\theta)=1$ in Theorem 2, we obtain Theorem 1. Similarly, taking $\omega(\theta)=1, \mathcal{F}(\theta)=\theta$ and $\Psi(\mathcal{F}(\theta))=\frac{\theta^{\kappa}}{\Gamma(\kappa)}$, we obtain the result of Dahmani [27]. 


\section{Generalized Weighted-Type Integral Inequalities Associated with Weighted and Extended Chebyshev Functionals}

In this section, we construct certain weighted-type generalized fractional integral inequalities.

Theorem 3. If the two functions $\mathcal{Z}_{1}$ and $\mathcal{Z}_{2}$ are differentiable on $[0, \infty), \hbar_{1}$ is a positive and integrable function on $[0, \infty)$. Let $\mathcal{F}$ be positive and increasing on $[0, \infty[$ and its derivative be continuous on $\left[0, \infty\left[\right.\right.$. If $\mathcal{Z}_{1}^{\prime} \in \mathcal{L}^{p}\left(\left[0, \infty[), \mathcal{Z}_{2}^{\prime} \in \mathcal{L}^{q}\left(\left[0, \infty[), p, q, r>1\right.\right.\right.\right.$ with $\frac{1}{p}+\frac{1}{p^{\prime}}=1$, $\frac{1}{q}+\frac{1}{q^{\prime}}=1$ and $\frac{1}{r}+\frac{1}{r^{\prime}}=1$, then the following weighted fractional integral inequality holds:

$$
\begin{aligned}
& 2\left|\left({ }_{\omega}^{\mathcal{F}} \mathcal{I}_{v_{1}+}^{\Psi} \hbar_{1}\right)(\theta)\left({ }_{\omega}^{\mathcal{F}} \mathcal{I}_{v_{1}+}^{\Psi} \hbar_{1} \mathcal{Z}_{1} \mathcal{Z}_{2}\right)(\theta)-\left({ }_{\omega}^{\mathcal{F}} \mathcal{I}_{v_{1}+}^{\Psi} \hbar_{1} \mathcal{Z}_{1}\right)(\theta)\left({ }_{\omega}^{\mathcal{F}} \mathcal{I}_{v_{1}+}^{\Psi} \hbar_{1} \mathcal{Z}_{2}\right)(\theta)\right| \\
& \leq\left(\left\|\mathcal{Z}_{1}^{\prime}\right\|_{p}^{r} \omega^{-r}(\theta) \int_{v_{1}}^{\theta} \int_{v_{1}}^{\theta} \frac{\Psi(\mathcal{F}(\theta)-\mathcal{F}(\varrho))}{\mathcal{F}(\theta)-\mathcal{F}(\varrho)} \frac{\Psi(\mathcal{F}(\theta)-\mathcal{F}(\zeta))}{\mathcal{F}(\theta)-\mathcal{F}(\zeta)} \omega(\zeta) \mathcal{F}^{\prime}(\zeta) \mathcal{F}^{\prime}(\zeta) \omega(\zeta) \hbar_{1}(\varrho) \hbar_{1}(\zeta)\right. \\
&\left.\times|\varrho-\zeta|^{\frac{1}{p^{\prime}}+\frac{1}{q^{\prime}}} d \varrho d \zeta\right)^{\frac{1}{r}} \times\left(\left\|\mathcal{Z}_{2}^{\prime}\right\|_{q}^{r^{\prime}} \omega^{-r^{\prime}}(\theta) \int_{v_{1}}^{\theta} \int_{v_{1}}^{\theta} \frac{\Psi(\mathcal{F}(\theta)-\mathcal{F}(\varrho))}{\mathcal{F}(\theta)-\mathcal{F}(\varrho)} \frac{\Psi(\mathcal{F}(\theta)-\mathcal{F}(\zeta))}{\mathcal{F}(\theta)-\mathcal{F}(\zeta)} \mathcal{F}^{\prime}(\varrho) \omega(\varrho) \mathcal{F}^{\prime}(\zeta) \omega(\zeta) \hbar_{1}(\varrho) \hbar_{1}(\zeta)\right. \\
&\left.\times|\varrho-\zeta|^{\frac{1}{p^{\prime}}+\frac{1}{q^{\prime}}} d \varrho d \zeta\right)^{\frac{1}{r^{\prime}}} \\
& \leq\left\|\mathcal{Z}_{1}^{\prime}\right\|_{p}\left\|\mathcal{Z}_{2}^{\prime}\right\|_{q} \omega^{-2}(\theta)\left(\int_{v_{1}}^{\theta} \int_{v_{1}}^{\theta} \frac{\Psi(\mathcal{F}(\theta)-\mathcal{F}(\varrho))}{\mathcal{F}(\theta)-\mathcal{F}(\varrho)} \frac{\Psi(\mathcal{F}(\theta)-\mathcal{F}(\zeta))}{\mathcal{F}(\theta)-\mathcal{F}(\zeta)} \mathcal{F}^{\prime}(\varrho) \omega(\varrho) \mathcal{F}^{\prime}(\zeta) \omega(\zeta) \hbar_{1}(\varrho) \hbar_{1}(\zeta)\right. \\
&\left.\times|\varrho-\zeta|^{\frac{1}{p^{\prime}}+\frac{1}{q^{\prime}}} d \varrho d \zeta\right) .
\end{aligned}
$$

Proof. Let us define

$$
\begin{aligned}
& H(\varrho, \zeta)=\left(\mathcal{Z}_{1}(\varrho)-\mathcal{Z}_{1}(\zeta)\right)\left(\mathcal{Z}_{2}(\varrho)-\mathcal{Z}_{1}(\zeta)\right) ; \varrho, \zeta \in\left(v_{1} \theta\right) \\
& \quad=\mathcal{Z}_{1}(\varrho) \mathcal{Z}_{2}(\varrho)-\mathcal{Z}_{1}(\varrho) \mathcal{Z}_{2}(\zeta)-\mathcal{Z}_{1}(\zeta) \mathcal{Z}_{2}(\varrho)+\mathcal{Z}_{1}(\zeta) \mathcal{Z}_{2}(\zeta) .
\end{aligned}
$$

Conducting the product of $(26)$ by $\omega^{-1}(\theta) \frac{\Psi(\mathcal{F}(\theta)-\mathcal{F}(\varrho))}{\mathcal{F}(\theta)-\mathcal{F}(\varrho)} \mathcal{F}^{\prime}(\varrho) \omega(\varrho) \hbar_{1}(\varrho)$, then integrating with respect to $\varrho$ over $\left(v_{1}, \theta\right)$ and using $(9)$, we obtain:

$$
\begin{aligned}
& \omega^{-1}(\theta) \int_{v_{1}}^{\theta} \frac{\Psi(\mathcal{F}(\theta)-\mathcal{F}(\varrho))}{\mathcal{F}(\theta)-\mathcal{F}(\varrho)} \mathcal{F}^{\prime}(\varrho) \omega(\varrho) \hbar_{1}(\varrho) H(\varrho, \zeta) d \varrho \\
= & \left({ }_{\omega}^{\mathcal{F}} \mathcal{I}_{v_{1}+}^{\Psi} \hbar_{1} \mathcal{Z}_{1} \mathcal{Z}_{2}\right)(\theta)-\mathcal{Z}_{2}(\zeta)\left({ }_{{ }_{\omega}}^{\mathcal{F}} \mathcal{I}_{v_{1}+}^{\Psi} \hbar_{1} \mathcal{Z}_{1}\right)(\theta)-\mathcal{Z}_{1}(\zeta)\left({ }_{\omega}^{\mathcal{F}} \mathcal{I}_{v_{1}+}^{\Psi} \hbar_{1} \mathcal{Z}_{2}\right)(\theta)+\mathcal{Z}_{1}(\zeta) \mathcal{Z}_{2}(\zeta)\left({ }_{\omega}^{\mathcal{F}} \mathcal{I}_{v_{1}+}^{\Psi} \hbar_{1}\right)(\theta) .
\end{aligned}
$$

Again, taking the product of $(27)$ by $\omega^{-1}(\theta) \frac{\Psi(\mathcal{F}(\theta)-\mathcal{F}(\zeta))}{\mathcal{F}(\theta)-\mathcal{F}(\zeta)} \mathcal{F}^{\prime}(\zeta) \omega(\zeta) \hbar_{1}(\zeta)$, then integrating with respect to $\zeta$ over $\left(v_{1}, \theta\right)$ and using (9), we have:

$$
\begin{aligned}
& \boldsymbol{\omega}^{-2}(\theta) \int_{v_{1}}^{\theta} \int_{v_{1}}^{\theta} \frac{\Psi(\mathcal{F}(\theta)-\mathcal{F}(\varrho))}{\mathcal{F}(\theta)-\mathcal{F}(\varrho)} \frac{\Psi(\mathcal{F}(\theta)-\mathcal{F}(\zeta))}{\mathcal{F}(\theta)-\mathcal{F}(\zeta)} \mathcal{F}^{\prime}(\varrho) \omega(\varrho) \hbar_{1}(\varrho) \mathcal{F}^{\prime}(\zeta) \omega(\zeta) \hbar_{1}(\zeta) H(\varrho, \zeta) d \varrho d \zeta \\
= & 2\left({ }_{\omega}^{\mathcal{F}} \mathcal{I}_{v_{1}+}^{\Psi} \hbar_{1}\right)(\theta)\left({ }_{\mathscr{\omega}} \mathcal{I}_{v_{1}+}^{\Psi} \hbar_{1} \mathcal{Z}_{1} \mathcal{Z}_{2}\right)(\theta)-\left({ }_{\omega}^{\mathcal{F}} \mathcal{I}_{v_{1}+}^{\Psi} \hbar_{1} \mathcal{Z}_{1}\right)(\theta)\left({ }_{\omega}^{\mathcal{F}} \mathcal{I}_{v_{1}+}^{\Psi} \hbar_{1} \mathcal{Z}_{2}\right)(\theta) .
\end{aligned}
$$

On the other side, we also have:

$$
H(\varrho, \zeta)=\int_{\zeta}^{\varrho} \int_{\zeta}^{\varrho} \mathcal{Z}_{1}^{\prime}(u) \mathcal{Z}_{2}^{\prime}(v) d u d v .
$$

By employing the Hölder inequality, we have:

$$
\left|\mathcal{Z}_{1}(\varrho)-\mathcal{Z}_{2}(\zeta)\right| \leq\left.\left.|\varrho-\zeta|^{\frac{1}{p^{\prime}}}\left|\int_{\zeta}^{\varrho}\right| \mathcal{Z}_{1}^{\prime}(u)\right|^{p} d u\right|^{\frac{1}{p}}
$$


and:

$$
\left|\mathcal{Z}_{2}(\varrho)-\mathcal{Z}_{2}(\zeta)\right| \leq\left.\left.|\varrho-\zeta|^{\frac{1}{q^{\prime}}}\left|\int_{\zeta}^{\varrho}\right| \mathcal{Z}_{2}^{\prime}(v)\right|^{q} d v\right|^{\frac{1}{q}} .
$$

Thus, $H$ can be estimated as:

$$
|H(\varrho, \zeta)| \leq\left.\left.\left.\left.|\varrho-\zeta|^{\frac{1}{p^{\prime}}+\frac{1}{q^{\prime}}}\left|\int_{\zeta}^{\varrho}\right| \mathcal{Z}_{1}^{\prime}(u)\right|^{p} d u\right|^{\frac{1}{p}}\left|\int_{\zeta}^{\varrho}\right| \mathcal{Z}_{2}^{\prime}(v)\right|^{q} d v\right|^{\frac{1}{q}} .
$$

Hence, from (28) and (32), it follows that:

$$
\begin{aligned}
& 2\left|\left({ }_{\omega}^{\mathcal{F}} \mathcal{I}_{v_{1}+}^{\Psi} \hbar_{1}\right)(\theta)\left({ }_{\omega}^{\mathcal{F}} \mathcal{I}_{v_{1}+}^{\Psi} \hbar_{1} \mathcal{Z}_{1} \mathcal{Z}_{2}\right)(\theta)-\left({ }_{\omega}^{\mathcal{F}} \mathcal{I}_{v_{1}+}^{\Psi} \hbar_{1} \mathcal{Z}_{1}\right)(\theta)\left({ }_{\omega} \mathcal{I}_{v_{1}+}^{\Psi} \hbar_{1} \mathcal{Z}_{2}\right)(\theta)\right| \\
= & \omega^{-2}(\theta) \int_{v_{1}}^{\theta} \int_{v_{1}}^{\theta} \frac{\Psi(\mathcal{F}(\theta)-\mathcal{F}(\varrho))}{\mathcal{F}(\theta)-\mathcal{F}(\varrho)} \frac{\Psi(\mathcal{F}(\theta)-\mathcal{F}(\zeta))}{\mathcal{F}(\theta)-\mathcal{F}(\zeta)} \mathcal{F}^{\prime}(\varrho) \omega(\varrho) \hbar_{1}(\varrho) \mathcal{F}^{\prime}(\zeta) \omega(\zeta) \hbar_{1}(\zeta)|H(\varrho, \zeta)| d \varrho d \zeta \\
\leq & \omega^{-2}(\theta) \int_{v_{1}}^{\theta} \int_{v_{1}}^{\theta} \frac{\Psi(\mathcal{F}(\theta)-\mathcal{F}(\varrho))}{\mathcal{F}(\theta)-\mathcal{F}(\varrho)} \frac{\Psi(\mathcal{F}(\theta)-\mathcal{F}(\zeta))}{\mathcal{F}(\theta)-\mathcal{F}(\zeta)} \mathcal{F}^{\prime}(\varrho) \omega(\varrho) \hbar_{1}(\varrho) \mathcal{F}^{\prime}(\zeta) \omega(\zeta) \hbar_{1}(\zeta) \\
\times & \left.\left.\left.\left.|\varrho-\zeta|^{\frac{1}{p^{\prime}}+\frac{1}{q^{\prime}}}\left|\int_{\zeta}^{\varrho}\right| \mathcal{Z}_{1}^{\prime}(u)\right|^{p} d u\right|^{\frac{1}{p}}\left|\int_{\zeta}^{\varrho}\right| \mathcal{Z}_{2}^{\prime}(v)\right|^{q} d v\right|^{\frac{1}{q}} d \varrho d \zeta .
\end{aligned}
$$

By employing the Hölder inequality for the double integral for (33), we obtain:

$$
\begin{aligned}
& 2\left|\left({ }_{\omega}^{\mathcal{F}} \mathcal{I}_{v_{1}+}^{\Psi} \hbar_{1}\right)(\theta)\left({ }_{\omega}^{\mathcal{F}} \mathcal{I}_{v_{1}+}^{\Psi} \hbar_{1} \mathcal{Z}_{1} \mathcal{Z}_{2}\right)(\theta)-\left({ }_{\omega}^{\mathcal{F}} \mathcal{I}_{v_{1}+}^{\Psi} \hbar_{1} \mathcal{Z}_{1}\right)(\theta)\left({ }_{\omega}^{\mathcal{F}} \mathcal{I}_{v_{1}+}^{\Psi} \hbar_{1} \mathcal{Z}_{2}\right)(\theta)\right| \\
\leq & \leq \omega^{-2}(\theta)\left(\int_{v_{1}}^{\theta} \int_{v_{1}}^{\theta} \frac{\Psi(\mathcal{F}(\theta)-\mathcal{F}(\varrho))}{\mathcal{F}(\theta)-\mathcal{F}(\varrho)} \frac{\Psi(\mathcal{F}(\theta)-\mathcal{F}(\zeta))}{\mathcal{F}(\theta)-\mathcal{F}(\zeta)} \mathcal{F}^{\prime}(\varrho) \omega(\varrho) \hbar_{1}(\varrho) \mathcal{F}^{\prime}(\zeta) \omega(\zeta) \hbar_{1}(\zeta)\right. \\
\times & \left.\left.\left.|\varrho-\zeta|^{\frac{1}{p^{\prime}}+\frac{1}{q^{\prime}}}\left|\int_{\zeta}^{\varrho}\right| \mathcal{Z}_{1}^{\prime}(u)\right|^{p} d u\right|^{\frac{r}{p}} d \varrho d \zeta\right)^{\frac{1}{r}} \\
\times & \left(\int_{v_{1}}^{\theta} \int_{v_{1}}^{\theta} \frac{\Psi(\mathcal{F}(\theta)-\mathcal{F}(\varrho))}{\mathcal{F}(\theta)-\mathcal{F}(\varrho)} \frac{\Psi(\mathcal{F}(\theta)-\mathcal{F}(\zeta))}{\mathcal{F}(\theta)-\mathcal{F}(\zeta)} \mathcal{F}^{\prime}(\varrho) \omega(\varrho) \hbar_{1}(\varrho) \mathcal{F}^{\prime}(\zeta) \omega(\zeta) \hbar_{1}(\zeta)\right. \\
\times & \left.\left.\left.|\varrho-\zeta|^{\frac{1}{p^{\prime}}+\frac{1}{q^{\prime}}}\left|\int_{\zeta}^{\varrho}\right| \mathcal{Z}_{2}^{\prime}(v)\right|^{q} d v\right|^{\frac{r^{\prime}}{q}} d \varrho d \zeta\right)^{\frac{1}{r^{\prime}}} .
\end{aligned}
$$

Now, utilizing the following relations:

$$
\left.\left|\int_{\zeta}^{\varrho}\right| \mathcal{Z}_{1}^{\prime}(u)\right|^{p} d u \mid \leq\left\|\mathcal{Z}_{1}^{\prime}\right\|_{p}^{p} \text { and }\left.\left|\int_{\zeta}^{\varrho}\right| \mathcal{Z}_{2}^{\prime}(v)\right|^{q} d v \mid \leq\left\|\mathcal{Z}_{2}^{\prime}\right\|_{q}^{q},
$$

then (34) becomes,

$$
\begin{aligned}
& 2\left|\left({ }_{\omega}^{\mathcal{F}} \mathcal{I}_{v_{1}+}^{\Psi} \hbar_{1}\right)(\theta)\left({ }_{\omega}^{\mathcal{F}} \mathcal{I}_{v_{1}+}^{\Psi} \hbar_{1} \mathcal{Z}_{1} \mathcal{Z}_{2}\right)(\theta)-\left({ }_{\omega}^{\mathcal{F}} \mathcal{I}_{v_{1}+}^{\Psi} \hbar_{1} \mathcal{Z}_{1}\right)(\theta)\left({ }_{\omega} \mathcal{I}_{v_{1}+}^{\Psi} \hbar_{1} \mathcal{Z}_{2}\right)(\theta)\right| \\
\leq & \left(\left\|\mathcal{Z}_{1}^{\prime}\right\|_{p}^{r} \omega^{-r}(\theta) \int_{v_{1}}^{\theta} \int_{v_{1}}^{\theta} \frac{\Psi(\mathcal{F}(\theta)-\mathcal{F}(\varrho))}{\mathcal{F}(\theta)-\mathcal{F}(\varrho)} \frac{\Psi(\mathcal{F}(\theta)-\mathcal{F}(\zeta))}{\mathcal{F}(\theta)-\mathcal{F}(\zeta)} \mathcal{F}^{\prime}(\varrho) \omega(\varrho) \hbar_{1}(\varrho) \mathcal{F}^{\prime}(\zeta) \omega(\zeta) \hbar_{1}(\zeta)\right. \\
\times & \left.|\varrho-\zeta|^{\frac{1}{p^{+}}+\frac{1}{q^{\prime}}} d \varrho d \zeta\right)^{\frac{1}{r}}\left(\left\|\mathcal{Z}_{2}^{\prime}\right\|_{p}^{r^{\prime}} \omega^{-r^{\prime}}(\theta) \int_{v_{1}}^{\theta} \int_{v_{1}}^{\theta}\right. \\
\times & \left.\mathcal{F}^{\prime}(\varrho) \omega(\varrho) \hbar_{1}(\varrho) \mathcal{F}^{\prime}(\zeta) \omega(\zeta) \hbar_{1}(\zeta)|\varrho-\zeta|^{\frac{1}{p^{\prime}}+\frac{1}{q^{\prime}}} d \varrho d \zeta\right)^{\frac{1}{r^{\prime}}} .
\end{aligned}
$$

From (36), we have:

$$
\begin{aligned}
& 2\left|\left({ }_{\omega}^{\mathcal{F}} \mathcal{I}_{v_{1}+}^{\Psi} \hbar_{1}\right)(\theta)\left({ }_{\omega}^{\mathcal{F}} \mathcal{I}_{v_{1}+}^{\Psi} \hbar_{1} \mathcal{Z}_{1} \mathcal{Z}_{2}\right)(\theta)-\left({ }_{\omega}^{\mathcal{F}} \mathcal{I}_{v_{1}+}^{\Psi} \hbar_{1} \mathcal{Z}_{1}\right)(\theta)\left({ }_{\omega}^{\mathcal{F}} \mathcal{I}_{v_{1}+}^{\Psi} \hbar_{1} \mathcal{Z}_{2}\right)(\theta)\right| \\
& \leq\left\|\mathcal{Z}_{1}^{\prime}\right\|_{p}\left\|\mathcal{Z}_{2}^{\prime}\right\|_{q} \omega^{-2}(\theta) \int_{v_{1}}^{\theta} \int_{v_{1}}^{\theta} \frac{\Psi(\mathcal{F}(\theta)-\mathcal{F}(\varrho))}{\mathcal{F}(\theta)-\mathcal{F}(\varrho)} \frac{\Psi(\mathcal{F}(\theta)-\mathcal{F}(\zeta))}{\mathcal{F}(\theta)-\mathcal{F}(\zeta)} \\
& \times \mathcal{F}^{\prime}(\varrho) \omega(\varrho) \mathcal{F}^{\prime}(\zeta) \omega(\zeta) \hbar_{1}(\varrho) \hbar_{1}(\zeta)|\varrho-\zeta|^{\frac{1}{p^{\prime}}+\frac{1}{q^{\prime}}} d \varrho d \zeta,
\end{aligned}
$$


which completes the required result.

If we consider $\mathfrak{\omega}(\theta)=1$ in Theorem 3, the following new result can be obtained.

Corollary 3. If the two functions $\mathcal{Z}_{1}$ and $\mathcal{Z}_{2}$ are differentiable on $[0, \infty)$ and if $\hbar_{1}$ is integrable and a positive function on $[0, \infty)$, and we let $\mathcal{F}$ be a positive and increasing function on $[0, \infty[$ and its derivative be continuous on $\left[0, \infty\left[\right.\right.$, if $\mathcal{Z}_{1}^{\prime} \in \mathcal{L}^{p}\left(\left[0, \infty[), \mathcal{Z}_{2}^{\prime} \in \mathcal{L}^{q}([0, \infty[), p, q, r>1\right.\right.$ with $\frac{1}{p}+\frac{1}{p^{\prime}}=1, \frac{1}{q}+\frac{1}{q^{\prime}}=1$, and $\frac{1}{r}+\frac{1}{r^{\prime}}=1$, then the following inequality holds:

$$
\begin{aligned}
& 2\left|\left({ }^{\mathcal{F}} \mathcal{I}_{v_{1}+}^{\Psi} \hbar_{1}\right)(\theta)\left({ }^{\mathcal{F}} \mathcal{I}_{v_{1}+}^{\Psi} \hbar_{1} \mathcal{Z}_{1} \mathcal{Z}_{2}\right)(\theta)-\left({ }^{\mathcal{F}} \mathcal{I}_{v_{1}+}^{\Psi} \hbar_{1} \mathcal{Z}_{1}\right)(\theta)\left({ }^{\mathcal{F}} \mathcal{I}_{v_{1}+}^{\Psi} \hbar_{1} \mathcal{Z}_{2}\right)(\theta)\right| \\
\leq & \left(\left\|\mathcal{Z}_{1}^{\prime}\right\|_{p}^{r} \int_{v_{1}}^{\theta} \int_{v_{1}}^{\theta} \frac{\Psi(\mathcal{F}(\theta)-\mathcal{F}(\varrho))}{\mathcal{F}(\theta)-\mathcal{F}(\varrho)} \frac{\Psi(\mathcal{F}(\theta)-\mathcal{F}(\zeta))}{\mathcal{F}(\theta)-\mathcal{F}(\zeta)} \mathcal{F}^{\prime}(\varrho) \mathcal{F}^{\prime}(\zeta) \hbar_{1}(\varrho) \hbar_{1}(\zeta)\right. \\
\times & \left.|\varrho-\zeta|^{\frac{1}{p^{\prime}}+\frac{1}{q^{\prime}}} d \varrho d \zeta\right)^{\frac{1}{r}} \\
\times & \left(\left\|\mathcal{Z}_{2}^{\prime}\right\|_{q}^{r^{\prime}} \int_{v_{1}}^{\theta} \int_{v_{1}}^{\theta} \frac{\Psi(\mathcal{F}(\theta)-\mathcal{F}(\varrho))}{\mathcal{F}(\theta)-\mathcal{F}(\varrho)} \frac{\Psi(\mathcal{F}(\theta)-\mathcal{F}(\zeta))}{\mathcal{F}(\theta)-\mathcal{F}(\zeta)} \mathcal{F}^{\prime}(\varrho) \mathcal{F}^{\prime}(\zeta) \hbar_{1}(\varrho) \hbar_{1}(\zeta)\right. \\
\times & \left.|\varrho-\zeta|^{\frac{1}{p^{\prime}}+\frac{1}{q^{\prime}}} d \varrho d \zeta\right)^{\frac{1}{r^{\prime}}} \\
\leq & \left\|\mathcal{Z}_{1}^{\prime}\right\|_{p}\left\|\mathcal{Z}_{2}^{\prime}\right\|_{q}\left(\int_{v_{1}}^{\theta} \int_{v_{1}}^{\theta} \frac{\Psi(\mathcal{F}(\theta)-\mathcal{F}(\varrho))}{\mathcal{F}(\theta)-\mathcal{F}(\varrho)} \frac{\Psi(\mathcal{F}(\theta)-\mathcal{F}(\zeta))}{\mathcal{F}(\theta)-\mathcal{F}(\zeta)} \mathcal{F}^{\prime}(\varrho) \mathcal{F}^{\prime}(\zeta) \hbar_{1}(\varrho) \hbar_{1}(\zeta)\right. \\
\times & \left.|\varrho-\zeta|^{\frac{1}{p^{\prime}}+\frac{1}{q^{\prime}}} d \varrho d \zeta\right) .
\end{aligned}
$$

Remark 4. If we consider $\omega(\theta)=1, \mathcal{F}(\theta)=\theta$ and $\Psi(\mathcal{F}(\theta))=\frac{\theta^{\kappa}}{\Gamma(\kappa)}$ in Theorem 3, we arrive at the inequality established by Dahmani et al. [28].

Remark 5. Furthermore, if we consider $\omega=1, \mathcal{F}(\theta)=\theta$ and $\Psi(\mathcal{F}(\theta))=\theta$ in Theorem 3, then we obtain the inequality (3) on $[0, \theta]$.

Theorem 4. If the two functions $\mathcal{Z}_{1}$ and $\mathcal{Z}_{2}$ are differentiable on $[0, \infty)$ and if $\hbar_{1}$ and $\hbar_{1}^{\prime}$ are integrable and positive functions on $[0, \infty)$, we let $\mathcal{F}$ be positive and increasing on $[0, \infty[$ and its derivative be continuous on $\left[0, \infty\left[\right.\right.$, and iff $\mathcal{Z}_{1}^{\prime} \in \mathcal{L}^{p}\left(\left[0, \infty[), \mathcal{Z}_{2}^{\prime} \in \mathcal{L}^{q}([0, \infty[), p, q, r>1\right.\right.$ such that $\frac{1}{p}+\frac{1}{p^{\prime}}=1, \frac{1}{q}+\frac{1}{q^{\prime}}=1$ and $\frac{1}{r}+\frac{1}{r^{\prime}}=1$, then the following weighted fractional integral inequality holds:

$$
\begin{aligned}
& 2 \mid\left({ }_{\omega}^{\mathcal{F}} \mathcal{I}_{v_{1}+}^{\Psi} \hbar_{1}^{\prime}\right)(\theta)\left({ }_{\omega}^{\mathcal{F}} \mathcal{I}_{v_{1}+}^{\Psi} \hbar_{1} \mathcal{Z}_{1} \mathcal{Z}_{2}\right)(\theta)-\left({ }_{\omega}^{\mathcal{F}} \mathcal{I}_{v_{1}+}^{\Psi} \hbar_{1} \mathcal{Z}_{1}\right)(\theta)\left({ }_{\omega}^{\mathcal{F}} \mathcal{I}_{v_{1}+}^{\Psi} \hbar_{1}^{\prime} \mathcal{Z}_{2}\right)(\theta) \\
&-\left({ }_{\omega}^{\mathcal{F}} \mathcal{I}_{v_{1}+}^{\Psi} \hbar_{1}^{\prime} \mathcal{Z}_{1}\right)(\theta)\left({ }_{\omega}^{\mathcal{F}} \mathcal{I}_{v_{1}+}^{\Psi} \hbar_{1} \mathcal{Z}_{2}\right)(\theta)+\left({ }_{\omega}^{\mathcal{F}} \mathcal{I}_{v_{1}+}^{\Psi} \hbar_{1}^{\prime}\right)(\theta)\left({ }_{\omega}^{\mathcal{F}} \mathcal{I}_{v_{1}+}^{\Psi} \hbar_{1}^{\prime} \mathcal{Z}_{1} \mathcal{Z}_{2}\right)(\theta) \mid \\
& \leq\left(\left\|\mathcal{Z}_{1}^{\prime}\right\|_{p}^{r} \omega^{-r}(\theta) \int_{v_{1}}^{\theta} \int_{v_{1}}^{\theta} \frac{\Psi(\mathcal{F}(\theta)-\mathcal{F}(\varrho))}{\mathcal{F}(\theta)-\mathcal{F}(\varrho)} \frac{\Psi(\mathcal{F}(\theta)-\mathcal{F}(\zeta))}{\mathcal{F}(\theta)-\mathcal{F}(\zeta)} \mathcal{F}^{\prime}(\varrho) \omega(\varrho) \mathcal{F}^{\prime}(\zeta) \omega(\zeta) \hbar_{1}(\varrho) \hbar_{1}^{\prime}(\zeta)\right. \\
&\left.\times|\varrho-\zeta|^{\frac{1}{p^{\prime}}+\frac{1}{q^{\prime}}} d \varrho d \zeta\right)^{\frac{1}{r}} \times\left(\left\|\mathcal{Z}_{2}^{\prime}\right\|_{q}^{r^{\prime}} \omega^{-r^{\prime}}(\theta) \int_{v_{1}}^{\theta} \int_{v_{1}}^{\theta} \frac{\Psi(\mathcal{F}(\theta)-\mathcal{F}(\varrho))}{\mathcal{F}(\theta)-\mathcal{F}(\varrho)} \frac{\Psi(\mathcal{F}(\theta)-\mathcal{F}(\zeta))}{\mathcal{F}(\theta)-\mathcal{F}(\zeta)} \mathcal{F}^{\prime}(\varrho) \omega(\varrho) \mathcal{F}^{\prime}(\zeta) \omega(\zeta) \hbar_{1}(\varrho) \hbar_{1}^{\prime}(\zeta)\right. \\
&\left.\times|\varrho-\zeta|^{\frac{1}{p^{\prime}}+\frac{1}{q^{\prime}}} d \varrho d \zeta\right)^{\frac{1}{\gamma^{\prime}}} \\
& \leq \mid\left\|\mathcal{Z}_{1}^{\prime}\right\|_{p}\left\|\mathcal{Z}_{2}^{\prime}\right\|_{q} \omega^{-2}(\theta)\left(\int_{v_{1}}^{\theta} \int_{v_{1}}^{\theta} \frac{\Psi(\mathcal{F}(\theta)-\mathcal{F}(\varrho))}{\mathcal{F}(\theta)-\mathcal{F}(\varrho)} \frac{\Psi(\mathcal{F}(\theta)-\mathcal{F}(\zeta))}{\mathcal{F}(\theta)-\mathcal{F}(\zeta)} \mathcal{F}^{\prime}(\varrho) \omega(\varrho) \mathcal{F}^{\prime}(\zeta) \omega(\zeta) \hbar_{1}(\varrho) \hbar_{1}^{\prime}(\zeta)\right. \\
&\left.\times|\varrho-\zeta|^{\frac{1}{p^{\prime}}+\frac{1}{q^{\prime}}} d \varrho d \zeta\right) .
\end{aligned}
$$


Proof. Conducting the product of $(27)$ by $\omega^{-1}(\theta) \frac{\Psi(\mathcal{F}(\theta)-\mathcal{F}(\zeta))}{\mathcal{F}(\theta)-\mathcal{F}(\zeta)} \mathcal{F}^{\prime}(\zeta) \omega(\zeta) \hbar_{1}^{\prime}(\zeta)$, then integrating with respect to $\zeta$ over $\left(v_{1}, \theta\right)$ and using (9), we obtain:

$$
\begin{aligned}
& \omega^{-2}(\theta) \int_{v_{1}}^{\theta} \int_{v_{1}}^{\theta} \frac{\Psi(\mathcal{F}(\theta)-\mathcal{F}(\varrho))}{\mathcal{F}(\theta)-\mathcal{F}(\varrho)} \frac{\Psi(\mathcal{F}(\theta)-\mathcal{F}(\zeta))}{\mathcal{F}(\theta)-\mathcal{F}(\zeta)} \mathcal{F}^{\prime}(\varrho) \omega(\varrho) \hbar_{1}(\varrho) \mathcal{F}^{\prime}(\zeta) \omega(\zeta) \hbar_{1}^{\prime}(\zeta) H(\varrho, \zeta) d \varrho d \zeta \\
= & \left({ }_{\omega}^{\mathcal{F}} \mathcal{I}_{v_{1}+}^{\Psi} \hbar_{1}^{\prime}\right)(\theta)\left({ }_{\omega}^{\mathcal{F}} \mathcal{I}_{v_{1}+}^{\Psi} \hbar_{1} \mathcal{Z}_{1} \mathcal{Z}_{2}\right)(\theta)-\left({ }_{\omega}^{\mathcal{F}} \mathcal{I}_{v_{1}+}^{\Psi} \hbar_{1} \mathcal{Z}_{1}\right)(\theta)\left({ }_{\omega}^{\mathcal{F}} \mathcal{I}_{v_{1}+}^{\Psi} \hbar_{1}^{\prime} \mathcal{Z}_{2}\right)(\theta) \\
- & \left({ }_{\mathscr{\omega}}^{\mathcal{F}} \mathcal{I}_{v_{1}+}^{\Psi} \hbar_{1}^{\prime} \mathcal{Z}_{1}\right)(\theta)\left({ }^{\mathcal{F}} \mathcal{I}_{v_{1}+}^{\Psi} \hbar_{1} \mathcal{Z}_{2}\right)(\theta)+\left({ }_{\mathscr{\omega}}^{\mathcal{F}} \mathcal{I}_{v_{1}+}^{\Psi} \hbar_{1}^{\prime}\right)(\theta)\left({ }_{\omega}^{\mathcal{F}} \mathcal{I}_{v_{1}+}^{\Psi} \hbar_{1}^{\prime} \mathcal{Z}_{1} \mathcal{Z}_{2}\right)(\theta) .
\end{aligned}
$$

Using (32) in (37), we obtain:

$$
\begin{aligned}
& \mid\left({ }_{\omega}^{\mathcal{F}} \mathcal{I}_{v_{1}+}^{\Psi} \hbar_{1}^{\prime}\right)(\theta)\left({ }_{\omega}^{\mathcal{F}} \mathcal{I}_{v_{1}+}^{\Psi} \hbar_{1} \mathcal{Z}_{1} \mathcal{Z}_{2}\right)(\theta)-\left({ }_{\omega}^{\mathcal{F}} \mathcal{I}_{v_{1}+}^{\Psi} \hbar_{1} \mathcal{Z}_{1}\right)(\theta)\left({ }_{\omega}^{\mathcal{F}} \mathcal{I}_{v_{1}+}^{\Psi} \hbar_{1}^{\prime} \mathcal{Z}_{2}\right)(\theta) \\
- & \left({ }_{\omega}^{\mathcal{F}} \mathcal{I}_{v_{1}+}^{\Psi} \hbar_{1}^{\prime} \mathcal{Z}_{1}\right)(\theta)\left({ }_{\omega}^{\mathcal{F}} \mathcal{I}_{v_{1}+}^{\Psi} \hbar_{1} \mathcal{Z}_{2}\right)(\theta)+\left({ }_{\omega}^{\mathcal{F}} \mathcal{I}_{v_{1}+}^{\Psi} \hbar_{1}^{\prime}\right)(\theta)\left({ }_{\omega}^{\mathcal{F}} \mathcal{I}_{v_{1}+}^{\Psi} \hbar_{1}^{\prime} \mathcal{Z}_{1} \mathcal{Z}_{2}\right)(\theta) \mid \\
= & \boldsymbol{\omega}^{-2}(\theta) \int_{v_{1}}^{\theta} \int_{v_{1}}^{\theta} \frac{\Psi(\mathcal{F}(\theta)-\mathcal{F}(\varrho))}{\mathcal{F}(\theta)-\mathcal{F}(\varrho)} \frac{\Psi(\mathcal{F}(\theta)-\mathcal{F}(\zeta))}{\mathcal{F}(\theta)-\mathcal{F}(\zeta)} \mathcal{F}^{\prime}(\varrho) \omega(\varrho) \hbar_{1}(\varrho) \mathcal{F}^{\prime}(\zeta) \omega(\zeta) \hbar_{1}(\zeta)|H(\varrho, \zeta)| d \varrho d \zeta \\
\leq & \omega^{-2}(\theta) \int_{v_{1}}^{\theta} \int_{v_{1}}^{\theta} \frac{\Psi(\mathcal{F}(\theta)-\mathcal{F}(\varrho))}{\mathcal{F}(\theta)-\mathcal{F}(\varrho)} \frac{\Psi(\mathcal{F}(\theta)-\mathcal{F}(\zeta))}{\mathcal{F}(\theta)-\mathcal{F}(\zeta)} \mathcal{F}^{\prime}(\varrho) \omega(\varrho) \hbar_{1}(\varrho) \mathcal{F}^{\prime}(\zeta) \omega(\zeta) \hbar_{1}^{\prime}(\zeta) \\
\times & \left.\left.\left.\left.|\varrho-\zeta|^{\frac{1}{p^{\prime}}+\frac{1}{q^{\prime}}}\left|\int_{\zeta}^{\varrho}\right| \mathcal{Z}_{1}^{\prime}(u)\right|^{p} d u\right|^{\frac{1}{p}}\left|\int_{\zeta}^{\varrho}\right| \mathcal{Z}_{2}^{\prime}(v)\right|^{q} d v\right|^{\frac{1}{q}} d \varrho d \zeta .
\end{aligned}
$$

The desired proof can be easily obtained by applying a similar procedure as used in the proof of Theorem 3.

If we consider $\omega=1$ in Theorem 4 , then we obtain the following new result.

Corollary 4. If the two functions $\mathcal{Z}_{1}$ and $\mathcal{Z}_{2}$ are differentiable on $[0, \infty)$ and if $\hbar_{1}$ and $\hbar_{1}^{\prime}$ are integrable and positive functions on $[0, \infty)$, we let $\mathcal{F}$ be an increasing and positive function on $\left[0, \infty\left[\right.\right.$ and its derivative be continuous on $\left[0, \infty\left[\right.\right.$, and if $\mathcal{Z}_{1}^{\prime} \in \mathcal{L}^{p}\left(\left[0, \infty[), \mathcal{Z}_{2}^{\prime} \in \mathcal{L}^{q}([0, \infty[)\right.\right.$, $p, q, r>1$ such that $\frac{1}{p}+\frac{1}{p^{\prime}}=1, \frac{1}{q}+\frac{1}{q^{\prime}}=1$ and $\frac{1}{r}+\frac{1}{r^{\prime}}=1$, then the following fractional integral inequality holds:

$$
\begin{aligned}
& 2 \mid\left({ }^{\mathcal{F}} \mathcal{I}_{v_{1}+}^{\Psi} \hbar_{1}^{\prime}\right)(\theta)\left({ }^{\mathcal{F}} \mathcal{I}_{v_{1}+}^{\Psi} \hbar_{1} \mathcal{Z}_{1} \mathcal{Z}_{2}\right)(\theta)-\left({ }^{\mathcal{F}} \mathcal{I}_{v_{1}+}^{\Psi} \hbar_{1} \mathcal{Z}_{1}\right)(\theta)\left({ }^{\mathcal{F}} \mathcal{I}_{v_{1}+}^{\Psi} \hbar_{1}^{\prime} \mathcal{Z}_{2}\right)(\theta) \\
- & \left({ }^{\mathcal{F}} \mathcal{I}_{v_{1}+}^{\Psi} \hbar_{1}^{\prime} \mathcal{Z}_{1}\right)(\theta)\left({ }^{\mathcal{F}} \mathcal{I}_{v_{1}+}^{\Psi} \hbar_{1} \mathcal{Z}_{2}\right)(\theta)+\left({ }^{\mathcal{F}} \mathcal{I}_{v_{1}+}^{\Psi} \hbar_{1}^{\prime}\right)(\theta)\left({ }^{\mathcal{F}} \mathcal{I}_{v_{1}+}^{\Psi} \hbar_{1}^{\prime} \mathcal{Z}_{1} \mathcal{Z}_{2}\right)(\theta) \mid \\
\leq & \left(\left\|\mathcal{Z}_{1}^{\prime}\right\|_{p}^{r} \int_{v_{1}}^{\theta} \int_{v_{1}}^{\theta} \frac{\Psi(\mathcal{F}(\theta)-\mathcal{F}(\varrho))}{\mathcal{F}(\theta)-\mathcal{F}(\varrho)} \frac{\Psi(\mathcal{F}(\theta)-\mathcal{F}(\zeta))}{\mathcal{F}(\theta)-\mathcal{F}(\zeta)} \mathcal{F}^{\prime}(\varrho) \mathcal{F}^{\prime}(\zeta) \hbar_{1}(\varrho) \hbar_{1}^{\prime}(\zeta)\right. \\
\times & \left.|\varrho-\zeta|^{\frac{1}{p^{\prime}}+\frac{1}{q^{\prime}}} d \varrho d \zeta\right)^{\frac{1}{r}} \\
\times & \left(\left\|\mathcal{Z}_{2}^{\prime}\right\|_{q}^{r^{\prime}} \int_{v_{1}}^{\theta} \int_{v_{1}}^{\theta} \frac{\Psi(\mathcal{F}(\theta)-\mathcal{F}(\varrho))}{\mathcal{F}(\theta)-\mathcal{F}(\varrho)} \frac{\Psi(\mathcal{F}(\theta)-\mathcal{F}(\zeta))}{\mathcal{F}(\theta)-\mathcal{F}(\zeta)} \mathcal{F}^{\prime}(\varrho) \mathcal{F}^{\prime}(\zeta) \hbar_{1}(\varrho) \hbar_{1}^{\prime}(\zeta)\right. \\
& \left.\times|\varrho-\zeta|^{\frac{1}{p^{\prime}}+\frac{1}{q^{\prime}}} d \varrho d \zeta\right)^{\frac{1}{r^{\prime}}} \\
\leq & \left\|\mathcal{Z}_{1}^{\prime}\right\|_{p}\left\|\mathcal{Z}_{2}^{\prime}\right\|_{q}\left(\int_{v_{1}}^{\theta} \int_{v_{1}}^{\theta} \frac{\Psi(\mathcal{F}(\theta)-\mathcal{F}(\varrho))}{\mathcal{F}(\theta)-\mathcal{F}(\varrho)} \frac{\Psi(\mathcal{F}(\theta)-\mathcal{F}(\zeta))}{\mathcal{F}(\theta)-\mathcal{F}(\zeta)} \mathcal{F}^{\prime}(\varrho) \mathcal{F}^{\prime}(\zeta) \hbar_{1}(\varrho) \hbar_{1}^{\prime}(\zeta)\right. \\
& \left.\times|\varrho-\zeta|^{\frac{1}{p^{\prime}}+\frac{1}{q^{\prime}}} d \varrho d \zeta\right) .
\end{aligned}
$$

Remark 6. By considering $\hbar_{1}^{\prime}(\theta)=\hbar_{1}(\theta)$ in Theorem 4, we obtain Theorem 3.

Remark 7. If we consider $\omega(\theta)=1, \mathcal{F}(\theta)=\theta$ and $\Psi(\mathcal{F}(\theta))=\frac{\theta^{\kappa}}{\Gamma(\kappa)}$ in Theorem 4, then we are led to the result of Dahmani [28]. 


\section{Concluding Remarks}

By utilizing the proposed weighted-type generalized fractional integral operator, we established a class of new integral inequalities for differentiable functions related to Chebyshev's, weighted Chebyshev's, and extended Chebyshev's functionals. The obtained inequalities are in more general form than the existing inequalities, which have been published earlier in the literature. Our result's exceptional cases can be found in $[5,11,12,27-30]$. Furthermore, for other types of operators addressed in Remarks 1 and 2, certain new integral inequalities connected to Chebyshev's functional and its extensions given in the literature can be easily obtained. One may investigate certain other types of integral inequalities by employing the proposed operators in the near future.

Author Contributions: Conceptualization, G.R. and A.H.; methodology, G.R.; software, A.H.; validation, G.R., A.A. and K.S.N.; formal analysis, G.R., A.H. and K.S.N.; investigation, A.H.; resources, K.S.N. and R.N.M.; writing—original draft preparation, G.R., A.H. and K.S.N.; writing-review and editing, G.R., K.S.N. and R.N.M.; visualization, K.S.N.; supervision, G.R.; project administration, G.R. and K.S.N.; funding acquisition, R.N.M. All authors have read and agreed to the published version of the manuscript.

Funding: This research received no external funding.

Institutional Review Board Statement: Not Applicable.

Informed Consent Statement: Not Applicable.

Data Availability Statement: Not Applicable.

Acknowledgments: This work was supported by Taif University researchers supporting Project Number (TURSP-2020/102), Taif University, Taif, Saudi Arabia.

Conflicts of Interest: The authors declare that they have no competing interest.

\section{References}

1. Chebyshev, P.L. Sur les expressions approximatives des integrales definies par les autres prises entre les mêmes limites. Proc. Math. Soc. Charkov. 1882, 2, 93-98.

2. Cerone, P.; Dragomir, S.S. A refinement of the Gruss inequality and applications. Tamkang J. Math. 2007, 38, 37-49. [CrossRef]

3. Dragomir, S.S. A generalization of Gruss's inequality in inner product spaces and applications. J. Math. Anal. Appl. 1999, 237, 74-82. [CrossRef]

4. Mitrinović, D.S.; Pečarić, J.E.; Fink, A.M. Classical and New Inequalities in Analysis. In Mathematics and Its Applications (East European Series); Kluwer Academic Publishers Group: Dordrecht, The Netherlands, 1993; Volume 61, ISBN 0-7923-2064-6.

5. Dragomir, S.S. Some integral inequalities of Gruss type. Indian J. Pure Appl. Math. 2002, 31, 397-415. [CrossRef]

6. Anastassiou, G.; Hooshmandasl, M.R.; Ghasemi, A.; Moftakharzadeh, F. Montgomery identities for fractional integrals and related fractional inequalities. J. Inequal. Pure Appl. Math. 2009, 10, 1-6.

7. Belarbi, S.; Dahmani, Z. On some new fractional integral inequalities. J. Inequal. Pure Appl. Math. 2009, 10, 86.

8. Dahmani, Z.; Tabharit, L.; Taf, S. New inequalities via Riemann-Liouville fractional integration. Adv. Res. Sci. Comput. 2010, 2, 40-45.

9. Dahmani, Z.; Tabharit, L. On weighted Gruss type inequalities via fractional integration. J. Adv. Res. Pure Math. 2010, 2, 31-38. [CrossRef]

10. Sarikaya, M.Z.; Aktan, N.; Yildirim, H. On weighted Chebyshev-Grüss like inequalities on time scales. J. Math. Inequal. 2008, 2, 185-195. [CrossRef]

11. Tassaddiq, A.; Rahman, G.; Nisar, K.S.; Samraiz, M. Certain fractional conformable inequalities for the weighted and the extended Chebyshev functionals. Adv. Differ. Equ. 2020, 2020, 96. [CrossRef]

12. Rahman, G.; Ullah, Z.; Khan, A.; Set, E.; Nisar, K.S. Certain Chebyshev type inequalities involving fractional conformable integral operators. Mathematics 2019, 7, 364. [CrossRef]

13. Srivastava, S.M.; Kashuri, A.; Mohammed, P.O.; Nonlaopon, K. Certain Inequalities Pertaining to Some New Generalized Fractional Integral Operators. Fractal Fract. 2021, 5, 160. [CrossRef]

14. Elezovic, N.; Marangunic, L.; Pecaric, G. Some improvement of Güss type inequality. J. Math. Inequal. 2007, 1, 425-436. [CrossRef]

15. Dahmani, Z. New inequalities in fractional integrals. Int. J. Nonlinear Sci. 2010, 9, 493-497.

16. Mitrinovic, D.S. Analytic Inequalities; Springer: Berlin, Germany, 1970; Volume 61.

17. Sarikaya, M.Z.; Yildirim, H. On generalization of the Riesz potential. Indian J. Math. Math. Sci. 2007, 3, $231-235$.

18. Jarad, F.; Abdeljawad, T.; Shah, K. On the weighted fractional operators of a function with respect to another function. Fractals 2020, 28, 2040011. [CrossRef] 
19. Jarad, F.; Ugurlu, E.; Abdeljawad, T.; Baleanu, D. On a new class of fractional operators. Adv. Differ. Equ. 2017, $2017,247$. [CrossRef]

20. Khan, T.U.; Khan, M.A. Generalized conformable fractional integral operators. J. Comput. Appl. Math. 2019, 346, 378-389. [CrossRef]

21. Khalil, R.; Horani, M.A.; Yousef, A.; Sababheh, M. A new definition of fractional derivative. J. Comput. Appl. Math. 2014, 264, 6570. [CrossRef]

22. Mubeen, S.; Habibullah, G.M. k-Fractional integrals and application. Int. J. Contemp. Math. Sci. 2012, 7, 89-94. [CrossRef]

23. Sarikaya, M.Z.; Ertuğral, F. On the Generalized Hermite-Hadamard Inequalities. Ann. Univ. Craiova, Math. Comput. Sci. Ser. 2020, 47, 193-213.

24. Kilbas, A.A.; Sarivastava, H.M.; Trujillo, J.J. Theory and Application of Fractional Differential Equation; North Holland Mathematics Studies, Volume 204; Elsevier Science: Amsterdam, The Netherlands, 2006.

25. Samko, S.G.; Kilbas, A.A.; Marichev, O.I. Fractional Integrals and Derivatives, Theory and Applications; Nikol'ski, S.M., Ed.; Translated from the 1987 Russian Original, Revised by the Authors; Gordon and Breach Science Publishers: Yverdon, Switzerland, 1993.

26. Katugampola, U.N. Approach to a generalized fractional integral. Appl. Math. Comput. 2011, 218, 860-865.

27. Dahmani, Z. New inequalities for a class of differentiable functions. Int. J. Nonlinear Anal. Appl. 2011, 2, 19-23. [CrossRef]

28. Dahmani, Z.; Khameli, A.; Fareha, K. Some RL-integral inequalities for the weighted and the extended Chebyshev functionals. Konuralp J. Math. 2017, 5, 43-48.

29. Dahmani, Z. The Riemann-Liouville Operator to Generate Some New Inequalities. Int. J. Nonlinear Sci. 2011, 12, $452-455$.

30. Rahman, G.; Nisar, K.S.; Khan, S.U.; Baleanu, D.; Vijayakumar, V. On the weighted fractional integral inequalities for Chebyshev functionals. Adv. Differ. Equ. 2021, 2021, 18. [CrossRef] 